LA-UR- $8-7725$

Approved for public release;

distribution is unlimited.
Title: Subgrid models for mass and thermal diffusion in turbulent mixing

\author{
Author(s): H. Lim, Y. Yu, J. Glimm, X-L Li, Department of Applied \\ Mathematics \\ Stony Brook University \\ Stony Brook, NY 11794 \\ and D. H. Sharp \\ Los Alamos National Laboratory \\ Los Alamos, NM 87545 \\ Intended for: \\ Physics of Fluids
}

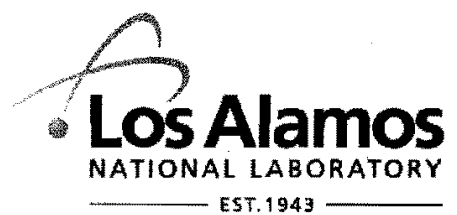

\begin{abstract}
Los Alamos National Laboratory, an affirmative action/equal opportunity employer, is operated by the Los Alamos National Security, LLC for the National Nuclear Security Administration of the U.S. Department of Energy under contract DE-AC52-06NA25396. By acceptance of this article, the publisher recognizes that the U.S. Government retains a nonexclusive, royalty-free license to publish or reproduce the published form of this contribution, or to allow others to do so, for U.S. Government purposes. Los Alamos National Laboratory requests that the publisher identify this article as work performed under the auspices of the U.S. Department of Energy. Los Alamos National Laboratory strongly supports academic freedom and a researcher's right to publish; as an institution, however, the Laboratory does not endorse the viewpoint of a publication or guarantee its technical correctness.
\end{abstract}




\title{
Subgrid models for mass and thermal diffusion in turbulent
}

\section{mixing*}

\author{
H. Lim, ${ }^{1}$ Y. Yu, ${ }^{1}$ J. Glimm $,{ }^{1}, 2$ X.-L. Li,${ }^{1}$ and D. H. Sharp ${ }^{3}$ \\ ${ }^{1}$ Department of Applied Mathematics and Statistics, \\ Stony Brook University, Stony Brook, NY 11794-3600, USA \\ ${ }^{2}$ Computational Science Center, Brookhaven National Laboratory, Upton, NY 11793-6000, USA \\ ${ }^{3}$ Los Alamos National Laboratory, Los Alamos, NM
}

(Dated: November 20, 2008)

We are concerned with the chaotic flow fields of turbulent mixing. Chaotic flow is found in an extreme form in multiply shocked Richtmyer-Meshkov unstable flows. The goal of a converged simulation for this problem is twofold: to obtain converged solutions for macro solution features, such as the trajectories of the principal shock waves, mixing zone edges, and mean densities and velocities within each phase, and also for such micro solution features as the joint probability distributions of the temperature and species concentration. We introduce parameterized subgrid models of mass and thermal diffusion, to define LES that replicate the micro features observed in the DNS. The Schmidt numbers and Prandtl numbers are chosen to represent typical liquid, gas and plasma parameter values. Our main result is to explore the variation of the Schmidt, Prandtl and Reynolds numbers by three orders of magnitude, and the mesh by a factor of 8 per linear dimension (up to 3200 cells per dimension), to allow exploration of both DNS and LES regimes and verification of the simulations for both macro and micro observables. We find mesh convergence for key properties describing the molecular level of mixing, including chemical reaction rates between the distinct fluid species. We find results nearly independent of Reynolds number for $R e 300,6000,600 K$.

Methodologically, the results are also new. In common with the shock capturing 
community, we allow and maintain sharp solution gradients, and we enhance these gradients through use of front tracking. In common with the turbulence modeling community, we include subgrid scale models with no adjustable parameters for LES. To the authors' knowledge, these two methodologies have not been previously combined. In contrast to both of these methodologies, our use of Front Tracking, with DNS or LES resolution of the momentum equation at or near the Kolmogorov scale, but without resolving the Batchelor scale, allows a feasible approach to the modeling of high Schmidt number flows.

PACS numbers: $47.27 . w j, 47.27 . \mathrm{tb}, 47.27 .-\mathrm{i}$

Keywords: Schmidt number, Prandtl number, Mass Diffusion, Turbulence, Multiphase Flow

\section{INTRODUCTION}

\section{A. Overview}

The turbulent mixing considered here is initiated a shock wave passing through a layer separating two fluids of distinct densities. When the layer is perturbed (or not normal relative to the shock wave), vorticity is deposited on the interface by the shock passage. This vorticity causes the interface to roll up and become unstable. Upon passage of a second shock wave, the interface enters an extremely chaotic regime. This is an example of a Richtmyer-Meshkov (RM) instability.

We consider a circular geometry, with a converging circular shock at the outer edge, and inside this, two fluids separated by a perturbed circular interface. The problem was previously described in detail ${ }^{15,16,19,30}$. The chaotic aspects of the mixing at a molecular level following reshock challenge some conventional ideas of computational science while supporting others. For this reason, the problem is of fundamental scientific interest, and 
may shed light on differing views for the computation of turbulent mixing flows.

Our goal is accurate numerical solutions using feasible grids. For the purpose, we validate the use of large eddy simulations (LES). Within this framework, we study mesh refinement and mesh convergence and dependence on a range of Reynolds numbers, including some within the regime of direct numerical simulation (DNS), in which transport properties are fully resolved. Initial and late time simulation density plots of unregularized simulations (zero transport coefficients) are shown in Fig. 1.

The fluid interface, at late time, is volume filling. The Reynolds number and transport coefficients (viscosity, mass diffusion, and heat conductivity) are given dimensionlessly as $R e=U L / \nu_{k}$, the Schmidt number $S c=\nu_{k} / D$, and the Prandtl number $\operatorname{Pr}=\nu_{k} / \alpha$. Here $\nu_{k}$ is the kinematic viscosity, $D$ the kinematic mass diffusivity and $\alpha=\frac{\kappa}{\rho c_{p}}$ the kinematic thermal diffusion rate. $\kappa$ is the heat conductivity, $\rho$ the density and $c_{p}$ the specific heat at constant pressure. $U$ and $L$ are characteristic velocity and length scales. Gases typically have Schmidt and Prandtl numbers of the order of unity, while liquids typically have Schmidt numbers in the range $S c \sim 4 \times 10^{2}$ to $10^{4}$ and Prandtl numbers in the range $\operatorname{Pr} \sim 10$ to $10^{2} \quad 1,22$. Dense plasma transport coefficients have been estimated as $S c \sim 0.6$ to $1.5^{25}$ for a range of plasma conditions relevant to NIF experiments. Plasma Prandtl numbers are dependent on ionization levels, nuclear charge, and temperature. A representative plasma value $\operatorname{Pr} \sim 10^{-4}$ can be inferred from the viscosity and heat conductivity values quoted by Drake $\mathrm{e}^{5}$. The very high level of thermal conductivity reflected in this value results from the transport of free electrons, and a time scale sufficient for the thermalization that allows a single temperature description of the plasma. We make no comments on this time scale. Numerical Schmidt and Prandtl numbers are generally not documented. If we assume a numerical shock width of $1.5 \Delta x$ and a numerical contact width of $5 \Delta x$ for untracked simulations, numerical transport coefficients in the range of 0.3 would be reasonable. On this basis, we consider the transport cases $1, \mathrm{~g}$, p from Table I. We allow physical parameters $(R e, S c, P r)$ to vary by three orders of magnitude, and explore mesh refinement up to 3200 zones per linear dimension. To keep 

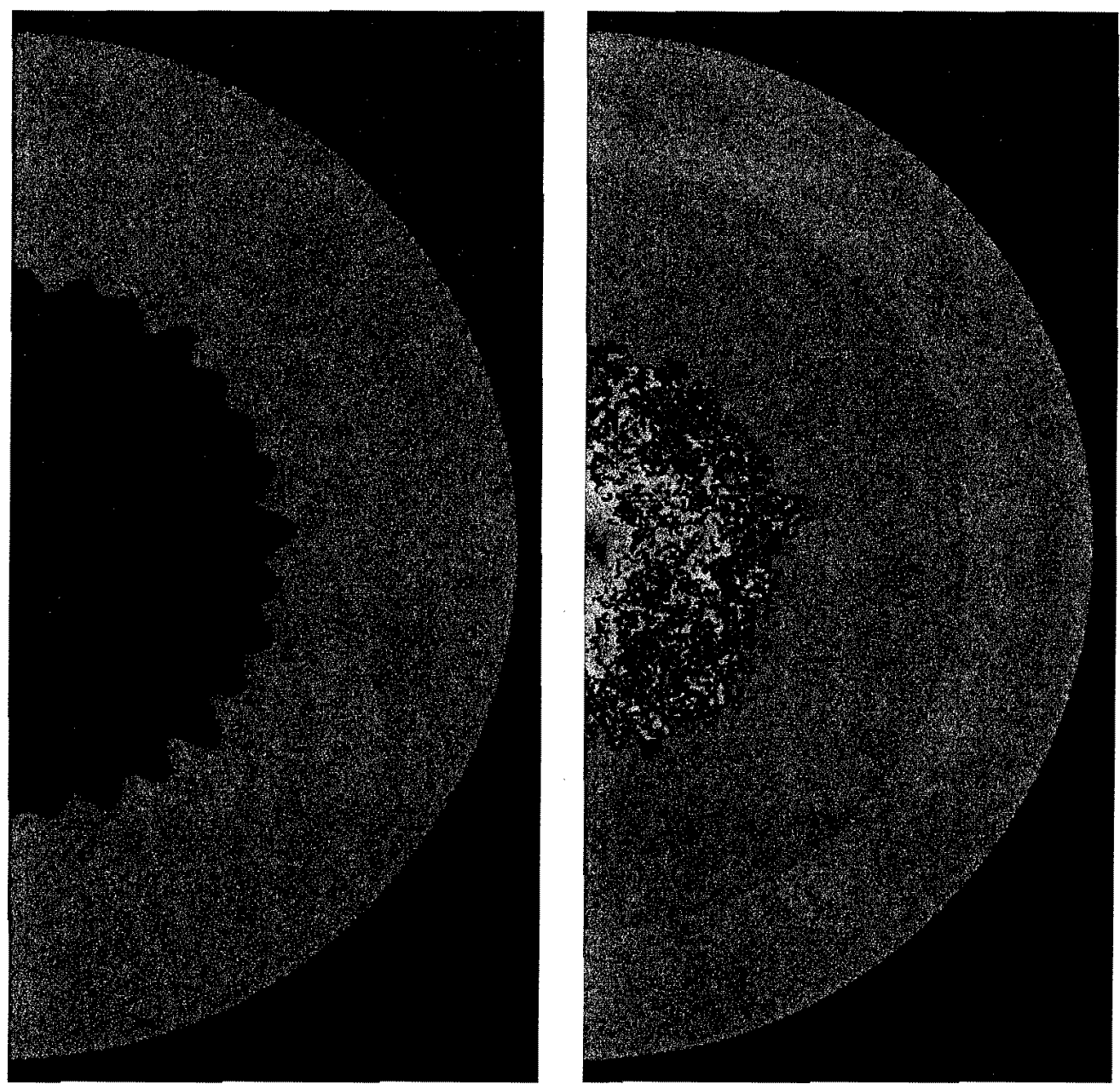

FIG. 1: Initial (left) and late time (right) density plot for the Richtmyer-Meshkov fluid instability (unregularized, with zero physical transport coefficients) under study in this paper.

the computational burden manageable, we restrict the simulations to $2 \mathrm{D}$ and we restrict the $2 \mathrm{D}$ mesh resolution to the finest resolution presently used for $3 \mathrm{D}$ simulations.

The emphasis on the joint probability distribution function (pdf) of concentration and temperature as micro scale observables is motivated by problems in turbulent combustion $^{21}$, where these variables affect the local flame speed and the overall flow.

The subgrid models are not original here, but their use in flow simulations having numerically sharp gradients appears to be new. In this sense, we are outside of the known 
TABLE I: Transport coefficients considered in this paper, arranged in order of increasing diffusivity. The presumed typical numerical transport parameters are shown for reference only and are not explored here.

\begin{tabular}{|c|c|c|}
\hline case & Schmidt & Prandtl \\
\hline 1 (liquid) & $10^{3}$ & 50 \\
\hline $\mathrm{g}(\mathrm{gas})$ & 1 & 1 \\
\hline $\mathrm{n}$ (numerical) & 0.3 & 0.3 \\
\hline p (plasma) & 1 & $10^{-4}$ \\
\hline
\end{tabular}

domain of validity of the subgrid models, and the present work serves as a verification study (i.e. mesh convergence and comparison to DNS).

At least for the present problem, with its somewhat modest number of initial modes, the joint pdf for concentration and temperature are subject to statistical fluctuations. In other words, the spatial averaging over the mixing zone is not sufficient to obtain statistical convergence, and an ensemble of simulations would be needed to obtain statistically converged mixing statistics. This can be observed from Fig. 1, where we see a significant variation in the size of the coherent mixing structures, and a relatively small sample of the larger sized ones, whose size appears to reflect the $t=0$ perturbation wave length. For this reason, we wish to introduce some degree of averaging into our analysis of the pdfs. Looking to the various applications in which turbulent mixing plays a role, chemical reactions stand out. The authors are involved in combustion modeling for scram jet design, type Ia supernova studies, and ICF motivated studies. Accordingly, we consider the chemical reaction rate $w$ of a hypothetical reaction $A+B \rightarrow C$ with a hypothetical activation temperature $T_{A C}$ to assess convergence of the pdfs. With this definition of convergence, the statistical fluctuations are reduced but not eliminated. Some degree of mesh convergence is observed, partially obscured by the remaining statistical fluctuations. 
To summarize, the main goal of this paper is to introduce and verify parameterized subgrid models for turbulent mass, momentum and thermal diffusion which will capture unresolved diffusive phenomena as it impacts coarse grid scales in a LES having steep numerical gradients. In this way, we plan to achieve LES which are converged relative to both the macro and the micro observables mentioned above. The simulations are more efficient than those of conventional turbulence models in the narrow width in mesh units that they allow for sharp gradient gradient concentration transitions that they allow. They model turbulent transport with microscopic observables correctly computed, in contrast to many capturing simulations. These subgrid models are applied here in a new context, namely to a front tracking and shock capturing Godunov scheme which maintains sharp gradients.

The front tracking code FronTier can achieve arbitrarily high Schmidt and Prandtl numbers numerically without a requirement for mesh refinement beyond that needed to resolve the turbulence, i.e. the vorticity field. This is not the case for most (untracked) simulation codes. Numerical mass and thermal diffusion arises primarily within the Eulerian hyperbolic step, due to solution averaging over grid blocks associated with transport by a nonzero velocity field (i.e. hyperbolic transport). This is apparently a universal feature of untracked Eulerian conservative capturing codes, but is circumvented with front tracking. Subgrid models can only add diffusion, and can never remove it. In this sense, a subgrid model for mass or thermal diffusion cannot cure the problem of excess numerical diffusion to achieve accurate modeling of atomic scale mixing. A conventional untracked code must be run in an "over resolved" mode, with more resolution than the momentum equation requires, to achieve low levels of numerical mass or thermal diffusion in the concentration and energy equations. In other words, conventional untracked codes require that the simulations be resolved or convergent in terms of the continuity, species and energy equations as well as for the momentum equation. The use of front tracking, however, avoids or reduces numerical diffusion in the species, continuity, and energy equations, even for coarse grids, and thus requires DNS or LES type convergence of the 
momentum equation alone.

We also point to the conclusions of Sec. IV, in which the macro variables (mixing zone edge positions, shock trajectories, etc.) are insensitive to physical or numerical modeling issues, and thus presumably to the choice of the subgrid model for viscosity, or to the viscosity and Reynolds number itself. Thus our convergence studies will focus primarily on the micro variables: the joint pdfs for species concentration and temperature and on the production rate for a typical chemical reaction. We will examine the degree to which these are insensitive to Reynolds number for LES, and to mesh for a fixed Reynolds number LES.

There is a large literature concerning turbulent mixing in RM unstable flows. Most of this literature focuses on macro observables, such as the mixing zone edges and shock trajectories. For RM mixing, these macro observables are insensitive, in that we find generically agreement among theory, experiment and numerics, $\mathrm{cf}^{13}{ }^{13}$

The micro observables (e.g. joint temperature and species PDFs) have received relatively less attention, but we can cite a previous study ${ }^{12}$, with conclusions distinct from ours, for a different RM flow. Among experimental studies of micro observables for RM instabilities, we mention two studies of related but distinct flow problems ${ }^{14,26}$. These experimental studies appear to be qualitatively consistent with our conclusions regarding the mixing, when compared at comparable times and Schmidt numbers. For example, quoting ${ }^{14}$, "Schmidt number plays a role in turbulent mixing of high-Reynolds flows".

The critical dependence of molecular levels of mixing on physical transport (and for under resolved capturing simulations, on numerical transport) is illustrated in Fig. 2. This dependence is one of the central points of this paper.

\section{EQUATIONS AND ALGORITHMS}

We study the compressible Navier-Stokes equations with viscosity, mass diffusion and thermal conductivity, for two miscible species initially separated by a sharp interface. The primitive equations describe the DNS limit, in which transport effects are resolved. 


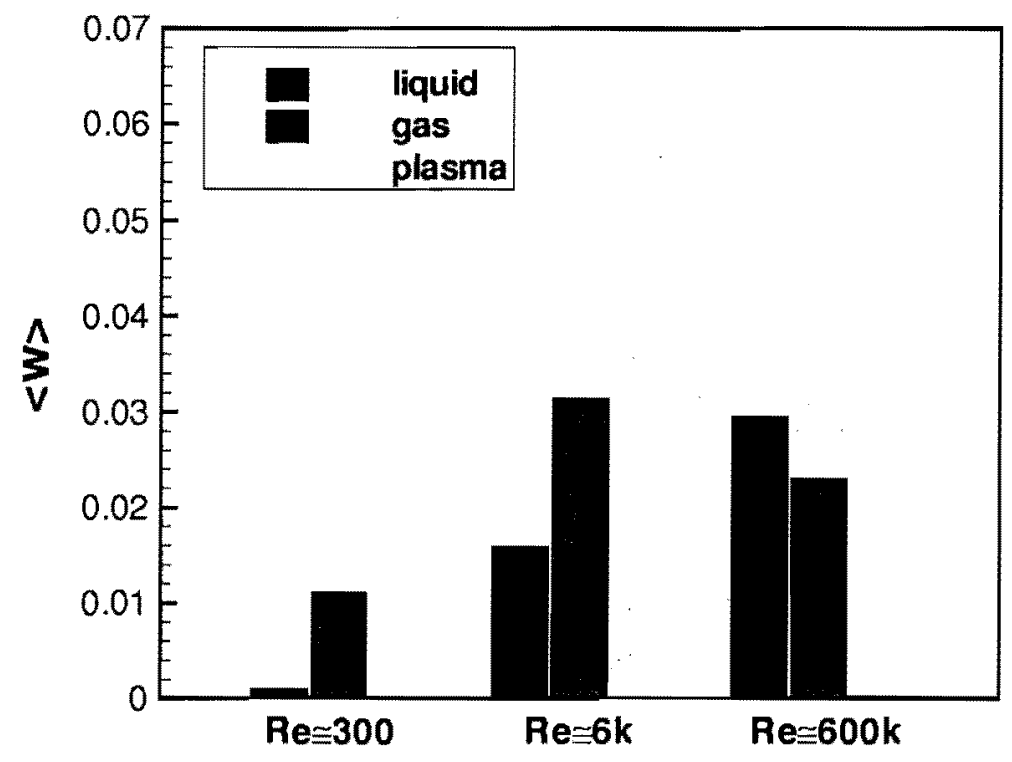

FIG. 2: Chemical reaction rate at $t=90$ for three representative values for the transport parameters, and each for three values of the Reynolds number. The activation temperature is chosen as $15,000^{\circ} \mathrm{K}$. The point of this plot is the strikingly different values obtained for $\langle w\rangle$ for each of the three physical regimes considered here.

A measure of this limit, as applied to the momentum equation, is the criteria $\lambda_{K \text { mesh }} \geq 1$ where $\lambda_{K \text { mesh }}=\lambda_{K} / \Delta x$ and $\lambda_{K}$ is the Kolmogorov length scale,

$$
\lambda_{K}=\left(\nu_{k}^{3} / \epsilon\right)^{1 / 4}
$$

where

$$
\epsilon=\nu_{k}|\mathbf{S}|^{2}
$$

$\mathbf{S}$ is the strain rate tensor

$$
S_{i j}=\frac{1}{2}\left(\frac{\partial v_{i}}{\partial x_{j}}+\frac{\partial v_{j}}{\partial x_{i}}\right)
$$

defined in terms of the velocity $\mathbf{v}$ and for any matrix $\mathbf{A}=A_{i j}$,

$$
|\mathbf{A}|^{2}=\sum 2 A_{i j}^{2}
$$


A related convergence measure, but applicable to all of the equations (not just to the momentum equation) is that the ratio of the turbulent transport parameters (i.e. the coefficients of the dynamic subgrid scale models (given below)) to the molecular ones be small.

LES start from a filter, or averaging procedure, applied to the primitive equations of compressible flow. We adopt what is known as an implicit filter, namely a grid block average. In this case the quantities in the defining equations are averaged over a grid block. New terms, arising from the average of the nonlinear terms, are introduced into the equations. We use a conventional definition of these terms, following refs. ${ }^{8,18,20}$. The subgrid models are parameterized dynamically, meaning that the model parameters are determined completely from the resolved scales. In this sense, the models are parameter free. For DNS, these terms have little effect.

We write the filtered continuity, momentum, energy and concentration equations for two miscible fluid species in an inertial frame. The filtered quantities are considered to be mesh block averages, and denoted with an overbar, while mass averaged quantities are denoted with a tilde. Repeated indices are summed.

$$
\begin{aligned}
& \frac{\partial \bar{\rho}}{\partial t}+\frac{\bar{\rho} \widetilde{v_{i}}}{\partial x_{i}}=0, \\
& \frac{\partial \bar{\rho} \widetilde{v_{j}}}{\partial t}+\frac{\partial\left(\bar{\rho} \widetilde{v_{i}} \widetilde{v_{j}}+\bar{p} \delta_{i j}\right)}{\partial x_{i}}=\frac{\partial \widetilde{d_{i j}}}{\partial x_{i}}-\frac{\partial \tau_{i j}}{\partial x_{i}}, \\
& \frac{\partial \bar{E}}{\partial t}+\frac{\partial(\bar{E}+\bar{p}) \widetilde{v_{i}}}{\partial x_{i}}=\frac{\partial \widetilde{d_{i j}} \widetilde{v_{j}}}{\partial x_{i}}+\frac{\partial}{\partial x_{i}}\left(\bar{\kappa} \frac{\partial \widetilde{T}}{\partial x_{i}}\right)+\frac{\partial}{\partial x_{i}}\left(\left(\widetilde{H_{h}}-\widetilde{H_{l}}\right) \bar{\rho} \widetilde{D} \frac{\partial \widetilde{\psi}}{\partial x_{i}}\right) \\
& +\left(\frac{1}{2} \frac{\partial \tau_{k k} \widetilde{v_{i}}}{\partial x_{i}}-\frac{\partial q_{i}^{(H)}}{\partial x_{i}}-\frac{\partial q_{i}^{(T)}}{\partial x_{i}}-\frac{\partial q_{i}^{(V)}}{\partial x_{i}}\right), \\
& \frac{\partial \bar{\rho} \widetilde{\psi}}{\partial t}+\frac{\partial \bar{\rho} \widetilde{\psi} \widetilde{v_{i}}}{\partial x_{i}}=\frac{\partial}{\partial x_{i}}\left(\widetilde{\rho} \widetilde{D} \frac{\partial \widetilde{\psi}}{\partial x_{i}}\right)-\frac{\partial q_{i}^{(\psi)}}{\partial x_{i}},
\end{aligned}
$$

where the subgrid scale (SGS) variables are the $\tau_{i j}, q_{i}^{(H)}, q_{i}^{(T)}, q_{i}^{(V)}$ and $q_{i}^{(\psi)}$. They are 
expressed as

$$
\begin{aligned}
\tau_{i j} & =\bar{\rho}\left(\widetilde{v_{i} v_{j}}-\widetilde{v_{i}} \widetilde{v_{j}}\right) \\
q_{i}^{(H)} & =\bar{\rho}\left(\widetilde{c_{p} T v_{i}}-\widetilde{c_{p}} \widetilde{T} \widetilde{v_{i}}\right) \\
q_{i}^{(T)} & =\frac{1}{2} \bar{\rho}\left(\widetilde{v_{k} v_{k} v_{i}}-\widetilde{v_{k}} \widetilde{v_{k}} \widetilde{v_{i}}\right) \\
q_{i}^{(V)} & =\overline{d_{i j} v_{j}}-\overline{d_{i j}} \widetilde{v_{j}} \\
q_{i}^{(\psi)} & =\bar{\rho}\left(\widetilde{\psi v_{i}}-\widetilde{\psi} \widetilde{v_{i}}\right) .
\end{aligned}
$$

The dependent filtered variables $\bar{\rho}, \widetilde{\psi}, \widetilde{v_{i}}, \bar{p}$ and $\bar{E}$ denote, respectively, the total mass density, the species mass fraction, the velocity, the pressure, and the total specific energy with

$$
\bar{E}=\bar{\rho} \widetilde{e}+\bar{\rho} \widetilde{v}_{k}^{2} / 2+\tau_{k k} / 2 .
$$

Here $\widetilde{H}_{h}$ and $\widetilde{H}_{l}$ are the partial specific enthalpy of each species defined by

$$
\begin{aligned}
& \widetilde{H}_{h}=\widetilde{e_{h}}+\frac{\bar{p}}{\bar{\rho}} \\
& \widetilde{H_{l}}=\widetilde{e_{l}}+\frac{\bar{p}}{\bar{\rho}},
\end{aligned}
$$

where $\widetilde{e_{h}}$ and $\widetilde{e_{l}}$ are the specific internal energy of each species. The equation of state for each of the species is taken to be a stiffened gamma law gas, and the mixture equation of state is also a stiffened gamma law gas ${ }^{2}$. This EOS is defined by two stiffening parameters, $e_{\infty}$ and $p_{\infty}$ and the adiabatic exponent $\gamma=c_{p} / c_{v}$. Here $c_{p}$ and $c_{v}$ are the specific heats at constant pressure and volume. For the mixture, $p_{\infty}$ is a volume average while $e_{\infty}, c_{p}$ and $c_{v}$ are mass weighted averages. $\gamma$ is the ratio of the mass weighted average $c_{p}$ and $c_{v}$.

The filtered internal energy $\widetilde{e}$ and the filtered pressure $\bar{p}$ are determined from the stiffened polytropic equation of state for a mixture of gases,

$$
\begin{gathered}
\tilde{e}=\widetilde{c_{v}} \widetilde{T}+\widetilde{e_{\infty}}+\frac{\overline{p_{\infty}}}{\bar{\rho}}+\widetilde{\left(\overline{c_{v} T}-\widetilde{c_{v}} \widetilde{T}\right),} \\
\bar{p}=(\widetilde{\gamma}-1) \bar{\rho} \widetilde{c_{v}} \widetilde{T}-\overline{p_{\infty}}+\bar{\rho}\left[\left(\widetilde{\gamma-1) c_{v}} T-(\widetilde{\gamma}-1) \widetilde{c_{v}} \widetilde{T}\right],\right.
\end{gathered}
$$


where $\widetilde{\gamma}$ is the adiabatic exponent, $\widetilde{c_{v}}$ is the specific heat at constant volume, $\widetilde{e_{\infty}}$ is the energy of formation and $\overline{p_{\infty}}$ has the dimensions of pressure.

For simplicity, we set the unclosed differences $\left.\widetilde{\left(\widetilde{c_{v} T}\right.}-\widetilde{c_{v}} \widetilde{T}\right)$ and $\left(\widetilde{\gamma-1) c_{v} T}-(\widetilde{\gamma}-1) \widetilde{c_{v}} \widetilde{T}\right.$ in (18) and (19) to zero. Having done this, the filtered EOS is a stiffened gamma law gas with filtered parameters $\widetilde{\gamma}, \widetilde{c_{v}}, \widetilde{e_{\infty}}$, and $\overline{p_{\infty}}$. Here the filtered parameters of this EOS are chosen following the same rule as for the mixture EOS defined above. Specifically

$$
\widetilde{\gamma}=\frac{\widetilde{c_{p}}}{\widetilde{c_{v}}}=\frac{c_{p, h} \widetilde{\psi}+c_{p, l}(1-\widetilde{\psi})}{c_{v, h} \widetilde{\psi}+c_{v, l}(1-\widetilde{\psi})},
$$

where the c's are the specific heat at constant pressure and volume and of the heavy and light fluids. Thus $\widetilde{\gamma}$ is not a Favre average of the $\gamma$ 's defining the mixture.

The viscous stress tensor $d_{i j}$ is expressed as

$$
\overline{d_{i j}}=\overline{\nu_{d}}\left(\left(\frac{\partial \widetilde{v_{i}}}{\partial x_{j}}+\frac{\partial \widetilde{v_{j}}}{\partial x_{i}}\right)-\frac{2}{3} \frac{\partial \widetilde{v_{k}}}{\partial x_{k}} \delta_{i j}\right)
$$

where $\overline{\nu_{d}}=\overline{\rho \nu_{k}}$ is the filtered dynamic viscosity. For simplicity, we take $\overline{\nu_{d}}$ to be a global constant. A more fundamental theory of multifluid viscosity is described in the book of Williams ${ }^{27}$.

To derive the filtered energy equation (7), we write

$$
\begin{aligned}
\overline{(E+p) v_{i}} & =\overline{\rho e v_{i}+\frac{1}{2} \rho v_{k} v_{k} v_{i}+p v_{i}} \\
& =\overline{\rho c_{v} T v_{i}+\rho e_{\infty} v_{i}+p_{\infty} v_{i}+(\gamma-1) \rho c_{v} T v_{i}-p_{\infty} v_{i}}+\frac{1}{2} \overline{\rho v_{k} v_{k} v_{i}} \\
& =\left(\bar{\rho} \widetilde{c_{v}} \widetilde{T} \widetilde{v_{i}}+\bar{\rho} \widetilde{e_{\infty}} \widetilde{v_{i}}+(\widetilde{\gamma}-1) \bar{\rho} \widetilde{c_{v}} \widetilde{T} \widetilde{v_{i}}+\overline{p_{\infty}} \widetilde{v_{i}}-\overline{p_{\infty}} \widetilde{v_{i}}\right)+\frac{1}{2} \bar{\rho} \widetilde{v_{k}} \widetilde{v_{k}} \widetilde{v_{i}} \\
& \left.+\bar{\rho}\left(\widetilde{\gamma c_{v} T v_{i}}-\widetilde{\gamma} \widetilde{c_{v}} \widetilde{T} \widetilde{v_{i}}\right)+\bar{\rho}\left(\widetilde{e_{\infty} v_{i}}-\widetilde{e_{\infty}} \widetilde{v_{i}}\right)+\frac{1}{2} \bar{\rho}\left(\widetilde{v_{k} v_{k} v_{i}}-\widetilde{v_{k}} \widetilde{v_{k}} \widetilde{v_{i}}\right)\right) \\
& =(\bar{E}+\bar{p}) \widetilde{v_{i}}+\left(q_{i}^{(H)}+q_{i}^{(T)}\right)-\frac{1}{2} \tau_{k k} \widetilde{v_{i}}+\bar{\rho}\left(\widetilde{e_{\infty} v_{i}}-\widetilde{e_{\infty}} \widetilde{v_{i}}\right) .
\end{aligned}
$$

We model the unclosed difference $\bar{\rho}\left(\widetilde{e_{\infty} v_{i}}-\widetilde{e_{\infty}} \widetilde{v_{i}}\right)$ as zero in (26).

The parabolic Navier-Stokes equations are solved via operator splitting, with separate solution steps for the hyperbolic and pure diffusion parts of the equations. The hyperbolic solutions are by the front tracking FronTier algorithm ${ }^{6}$. The interface hyperbolic updates are split into normal and tangential operators defined at front points. The 
normal update uses a predictor-corrector algorithm, with the predictor step a Riemann solution using the left and right front states as input. The corrector step, based on finite differences in characteristic coordinates, couples the normal direction front propagation to signals coming from interior states. The interior hyperbolic update uses a Godunov finite difference solver based on the MUSCL algorithm ${ }^{4,28}$. A sharp (tracked) interface in the hyperbolic update uses ghost cells ${ }^{11}$ in the interior state update to eliminate ${ }^{17}$ transport related numerical mass and thermal diffusion across the interface.

Physical transport for front states is introduced via finite differences in a normaltangential rectangular coordinate system at the front. Discretization is outlined in the appendix, where the turbulent transport terms are defined. The parabolic update of the interior states, i.e. the inclusion of transport terms for species diffusion, viscosity and thermal heat conductivity, is via conventional finite differences. Irregular stencils for the interior states, i.e. the stencils crossing the front, are treated conventionally, without regard to the front or any ghost cell values. An explicit solver for both the interior and the front state parabolic solvers, with possible time step subcycling, is sufficient to allow a stable computation for most of the mesh and transport parameter range considered.

For the highly heat diffusive plasma conditions, an implicit solver is used. With transport terms strong enough to require an implicit solver, there is no need for, or benefit from, tracking. Moreover, the formulation of the tracked front states together with the interior states appears to be inconsistent with common implicit solver algorithms. We allow selective untracking of specific (primitive) variables within an overall tracked solution. In this algorithm, after the interior and front state updates, the untracked front states (the primitive variables that are not being tracked, for example energy, in the case $p$ ) are re-determined by one sided interpolation from neighboring interior states of the same component. Here one sided means that the interpolating interior states are restricted to have the same component as that of the front state in question. In the unusual case of no nearby interior states with the required component, a two sided interpolation is used, in which the component restriction is dropped. As a test of this algorithm, we compared 
it to two sided interpolation, and we compared it to an explict algorithm (with tracking of the primitive energy variable). For this case, extensive subcycling in the parabolic step for the energy equation was needed for numerical stabiilty. There was no notable difference among these three algorithms.

The FronTier numerical Schmidt and Prandtl numbers are $\infty$, and the code allows efficient simulation of any desired (physical) Schmidt or Prandtl number.

\section{THE FLOW INSTABILITY PROBLEM}

In the problem considered here, see Fig. 1, the flow is dominated by a single strong shock wave, starting at the outer edge of the computational domain (a half circle). The shock passes through the interface separating the two fluids, proceeds to the origin, reflects there and expands outward, recrossing the interface region and finally exiting at the outer boundary. The interface between the two fluids is defined as a $50 \%$ isoconcentration contour.

The interface region, due to the shock induced instability, expands into a mixing zone, which has a very complex structure. Especially after the second passage of the shock (the reshock or reflected shock passage), the mixing zone becomes highly chaotic. The inner and outer edges of the mixing zone are defined in terms of $5 \%$ and $95 \%$ volume fraction contours, after a spatial average over the circular symmetry variable. The mixing zone is then defined as the region between these inner and outer edges. The software which captures the space time trajectory of these waves in the numerical solution is known as a wave filter ${ }^{7,9,30}$. A space time plot of the shock trajectories and mixing zone edges is shown in Fig. 3.

\section{MESH CONVERGENCE FOR MACRO OBSERVABLES}

We set a (time dependent) length scale $L$ to be the width of the mixing zone, and the velocity scale $U$ to be the turbulent velocity $U=\sqrt{\left\langle\delta v^{2}\right\rangle}$. The angle brackets $\langle\cdots\rangle$ 


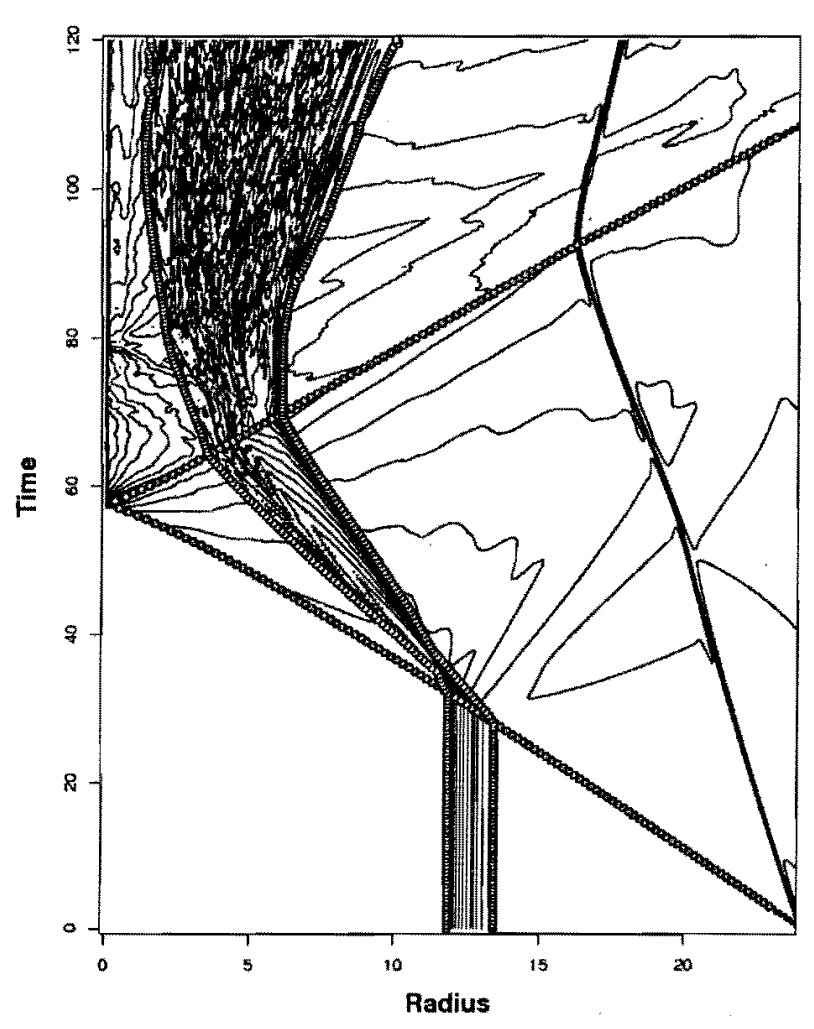

FIG. 3: Space time $(r, t)$ contours of the primary waves, as detected by the wave filter algorithm. These are the inward (direct) and outward (reflected) shock waves and the inner and outer edges of the mixing zone, all detected within a single rotational averaging window, in this case $\theta \in\left[-45^{\circ}, 0^{\circ}\right]$.

denote an angular (or in principle ensemble) average and $\delta v=v-\langle v\rangle$. We also define $R e_{\text {mesh }}=U \Delta x / \nu_{k}$.

The objective of the present section is to compute the large scale solution features accurately. This includes the trajectories of the principal waves, as illustrated in Fig. 3. This objective is related to a systematic convergence study ${ }^{15,30}$. In that study, we found statistical convergence for many mean flow variables, which define what we call the macroscopic description of the flow. In Fig. 4, we plot the time integrated relative wave discrepancy defined in terms of the mixing zone edge positions and the trajectories of 


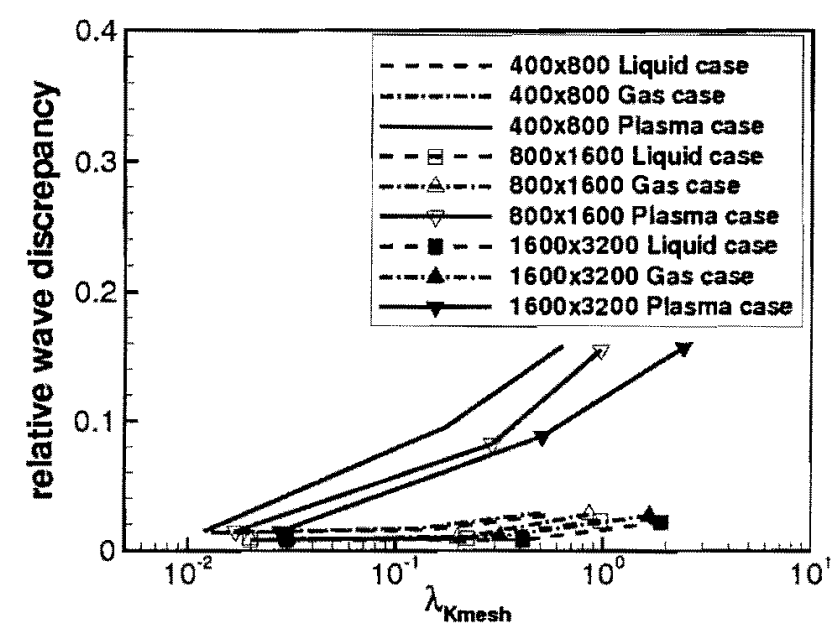

FIG. 4: Time integrated relative discrepancy in the mixing zone edge locations as compared to a fine grid, zero transport simulation. The discrepancy, for cases $l$ and $\mathrm{g}$, is mainly due to mesh errors and is partly due to modification of physical parameters. The discrepancy decreases with mesh refinement, uniformly as physical parameters are varied. The plasma case p shows convergence, but to a Reynolds number dependent limit distinct (for small $R e$ ) from the zero transport case.

the principal shock waves for a variety of mesh levels and for transport coefficients from Table I. Similar convergence properties have been obtained for other macro variable solution errors such as the mean densities and velocities for each phase. The error (or discrepancy) is determined by comparison of the simulation to a fine grid $(6400 \times 3200)$ simulation having zero transport coefficients. The reported discrepancy is thus a mixture of mesh errors and discrepancies associated with modification of the transport coefficients from a nominal value (zero).

From Fig. 4, and related studies ${ }^{15,19,30}$ we conclude that the macro observables are insensitive to both numerical and physical parameters, except for case $\mathrm{p}$ with low $R e$ (large transport parameters). 

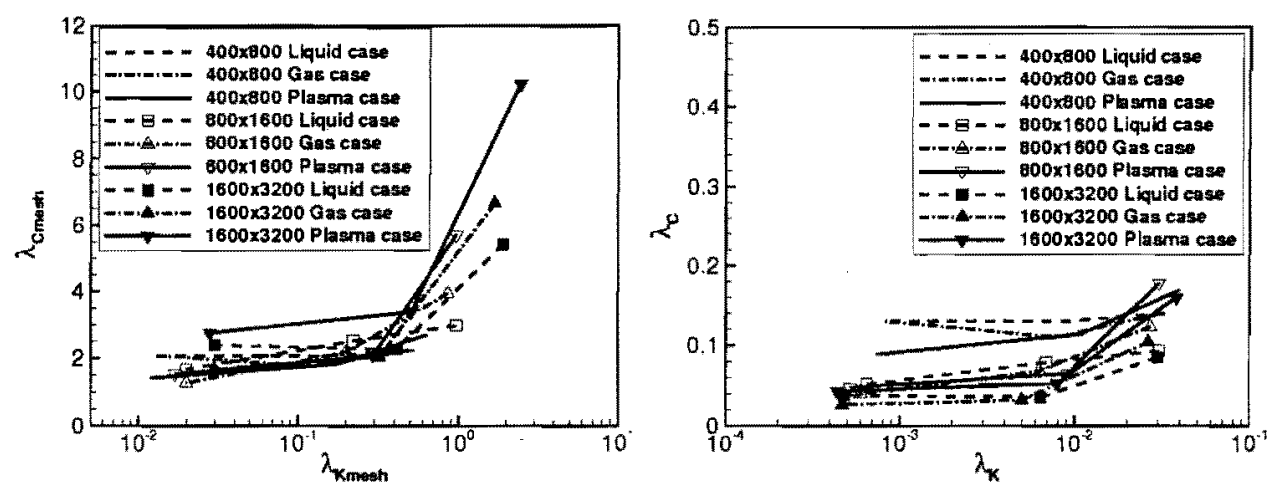

FIG. 5: Left: plot of $\lambda_{C} / \Delta x$ vs. $\lambda_{K \text { mesh }}$ for a range of mesh levels and transport parameters cases $\mathrm{l}, \mathrm{g}, \mathrm{p}$ from Table I, at $t=90, r=r_{\text {mid. }}$ Right: the same data replotted as $\lambda_{C}$ vs. $\lambda_{K}$.

\section{CORRELATION AND DIFFUSION LENGTH SCALES}

We introduce a correlation length scale $\lambda_{C}$ to characterize the microstructure of mix. The correlation length is defined in terms of the probability of exit distance $\xi$ from a given phase or mean distance to the complementary phase, introduced ${ }^{23,24}$ for models of opacity, and studied ${ }^{10,15,16}$ as a measure of fine scale mixing length. For random points situated on a radius $r$ within the mixing zone, the exit probability data is collected into bins, each holding the data for an interval of possible exit lengths. Using the probability measure $d \xi$, as defined by the binned data, we define $\lambda_{C}=\int \xi d \xi$. See Fig. 5 .

We assess interface convergence in terms of the behavior of $\lambda_{C}$. The DNS regime occurs to the right side of each frame of Fig. 5. In this regime, the right frame (with scales independent of $\Delta x$ ) suggests mesh convergence to a limit, with weak dependence on $S c$ and Pr. In the left frame of Fig. 5, we scale out the mesh dependence, and observe that $\lambda_{C \text { mesh }}$ is only weakly dependent on $\Delta x$ in the LES regime (left part of the frame). In other words, $\lambda_{C}$, for an LES simulation, is determined mainly by the mesh level.

We introduce the time dependent molecular diffusion length scales $\lambda_{D}=2(D(t-$ $\left.\left.t_{0}\right)\right)^{1 / 2}$, and $\lambda_{T}=2\left(\alpha\left(t-t_{0}\right)\right)^{1 / 2}\left(\alpha=\kappa / \rho c_{p}\right)$, where $t_{0}$ is the time of reshock. The corresponding turbulent length scales are $\lambda_{D_{t}}=2\left(D_{t}\left(t-t_{0}\right)\right)^{1 / 2}$, and $\lambda_{T_{t}}=2\left(\alpha_{t}(t-\right.$ 

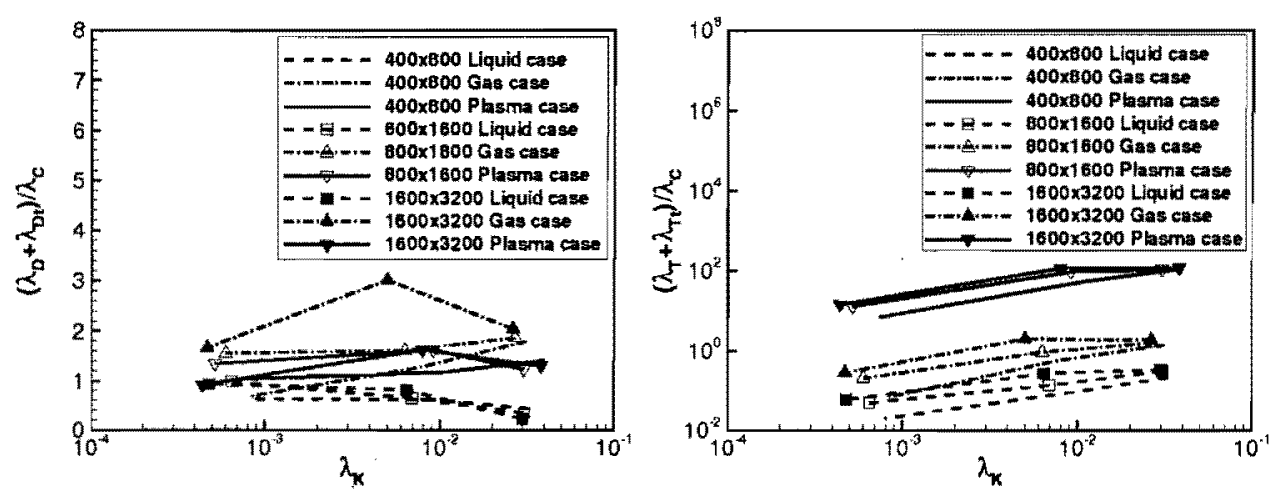

FIG. 6: The ratios $\left(\lambda_{D}+\lambda_{D_{t}}\right) / \lambda_{C}$ and $\left(\lambda_{T}+\lambda_{T_{t}}\right) / \lambda_{C}$ vs. $\lambda_{K}$ for several mesh levels and for transport coefficients cases $1, \mathrm{~g}, \mathrm{p}$ from Table I. The values to the far right in each frame are DNS, and appear to be mesh converged for the finer meshes.

$\left.\left.t_{0}\right)\right)^{1 / 2}$. Here $D_{t}=\nu_{k t} / S c_{t}$ and $\alpha_{t}=\nu_{k t} / P r_{t}$ are kinematic turbulent transport coefficients (species and temperature). $S c_{t}$ and $P r_{t}$ are turbulent transport coefficients and $\nu_{k t}$ is the kinematic turbulent viscosity, all defined in the appendix. The ratios $\left(\lambda_{D}+\lambda_{D_{t}}\right) / \lambda_{C}$ and $\left(\lambda_{T}+\lambda_{T_{t}}\right) / \lambda_{C}$ are dimensionless measures of the concentration and thermal mixing levels due to combined effects of molecular and turbulent transport. We plot these ratios vs. $\lambda_{K}$ in Fig. 6 for a variety of meshes and for transport coefficients from Table I. The large $\lambda_{K}$ asymptote (right side of each frame of Fig. 6) represents the DNS limit. This limit is converged to a grid independent value, which depends on $S c$ and $\operatorname{Pr}$.

The ratios are also mesh convergent for higher $R e$ (in the LES regime) and nearly independent of Re. We note an anomalous simulation for the fine grid gas case. Fig. 6 shows the success of the subgrid model, with the mesh converged LES simulations in excellent agreement with DNS. 


\section{CONVERGENCE OF MICRO OBSERVABLES: CONCENTRATION AND TEMPERATURE}

\section{A. Joint PDFs}

The joint pdf for the temperature and species mass concentrations of the fluid mixture is defined as a function of time and radius, assuming that the probability data is collected from the angular variation in space. To create the pdfs from the simulation data, we collect the temperature and concentration variables within the mixing zone. Mixed cells are not averaged, but each cell fraction contributes its own concentration fraction and temperature with its own probabilities (proportional to area). The concentration fractions and temperatures are then binned.

The liquid joint pdfs are bimodal. Both the liquid and the gas pdfs are concentrated near a curve in concentration-temperature space, joining the light to the heavy fluid concentrations. The origin of this shape can be explained by shock heating, which tends to heat the heavy fluid more strongly. This trend is reinforced by the initial conditions; initially the heavy fluid is hotter. Then portions of the heavy and light fluid diffuse into one another, so that the temperature pdf at fixed concentration is determined from the temperature pdf of the pure fluids before mixture through diffusion. The data presented is from the time $t=90$, which is the beginning of the chaotic stage of interface development.

We approach mesh convergence of the concentration-temperature statistics first through a study of the joint pdf. While the pdfs for common physics at three mesh levels are qualitatively similar and may be judged to show some signs of convergence, this trend is somewhat spotty. In fact the mixed region displays some number of large blobs of poorly mixed light material. The relative size of these regions is unstable, and an attempt to compare solutions (e.g. with distinct meshes) yields more variation than would be desired, at least at the present level of computation. We illustrate this fact in Table II.

The errors quoted are computed as $L_{1}$ norm differences in the joint probability density 


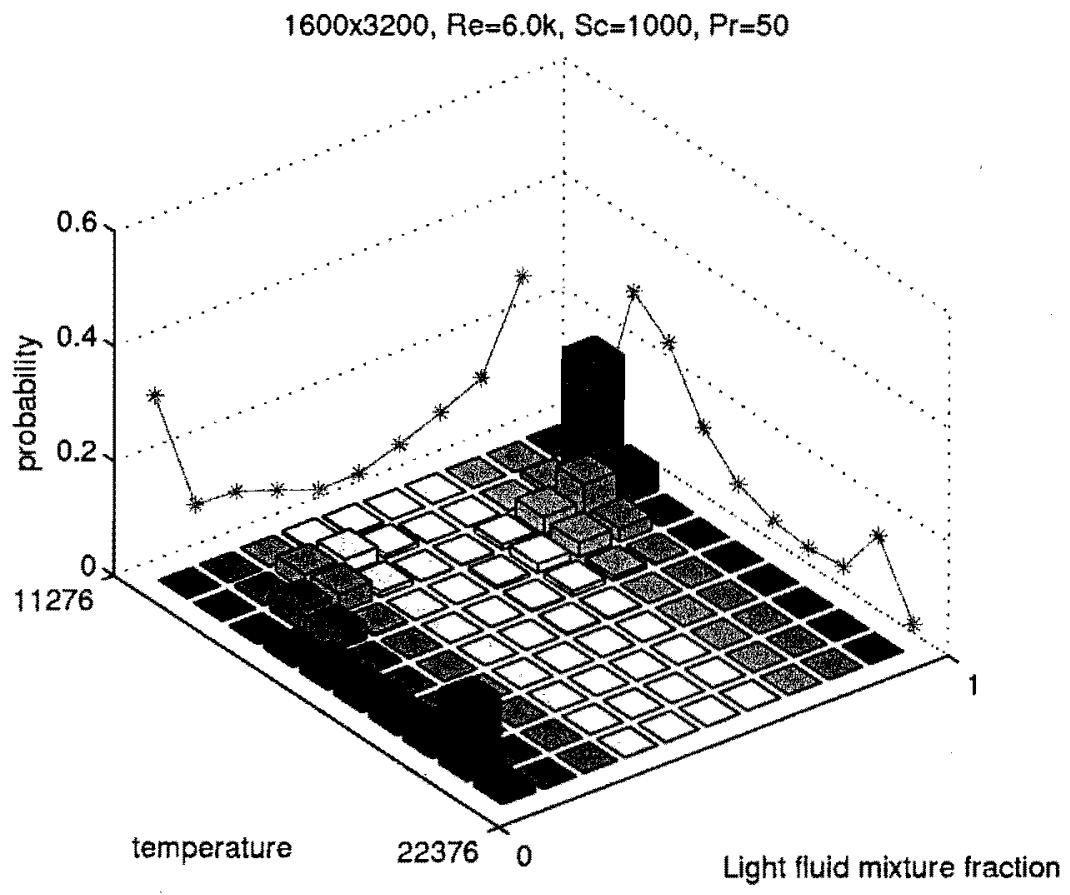

FIG. 7: Case 1. The joint pdf of light species concentration and temperature at time $t=90$. The data has been collected into $10 \times 10$ bins. The mesh is $1600 \times 3200$ and $R e \approx 6000$.

functions for species concentration and temperature. These pdf's are computed on a $10 \times 10$ range of values, with common end points for each of the meshes in the comparison. They are normalized so that the sum of the 100 individual values is 1 . We compute with three distinct meshes $(400 \times 800,800 \times 1600$ and $1600 \times 3200)$ for the cases $1, \mathrm{~g}$, and $\mathrm{p}$. Thus there are two levels of error for each case.

We define $u_{i j}$ to be the probability of $u$ for the bin $i, j$, which is the sum of observations of $u$ in bin $i, j$ divided by the total number of observations. With $u^{c}$ and $u^{f}$ denoting coarse and fine grid values, we have the the error formula

$$
e=\sum_{i, j=0}^{9}\left|u_{i, j}^{c}-u_{i, j}^{f}\right|
$$

for the pdf. 


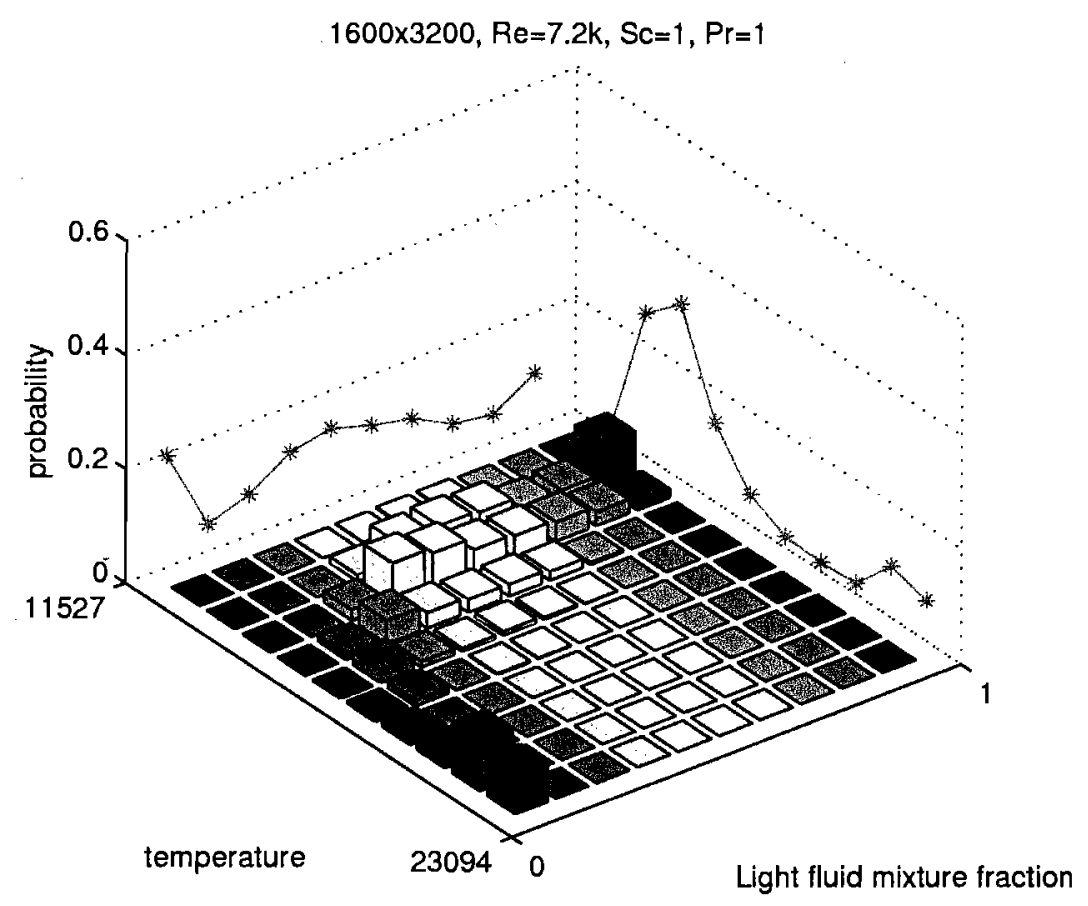

FIG. 8: Case g. The joint pdf of light species concentration and temperature at time $t=90$. The data has been collected into $10 \times 10$ bins. The mesh is $1600 \times 3200$ and $R e \approx 6000$.

The listed Reynolds numbers are approximate, and the refinements are performed (within each series of approximately constant Reynolds number) with identical molecular level transport coefficients. Thus the mesh refinement occurs within identically specified physics. The observed Reynolds numbers and the subgrid scale turbulent transport parameters are mesh dependent, and do vary within this comparison.

\section{B. Concentration Moments}

Although the errors in the pdfs decrease with mesh refinement, they are not uniformly small, and in the worst cases are as large as the pdfs themselves. For this reason, we next consider a more highly averaged analysis of the same statistical variables. 


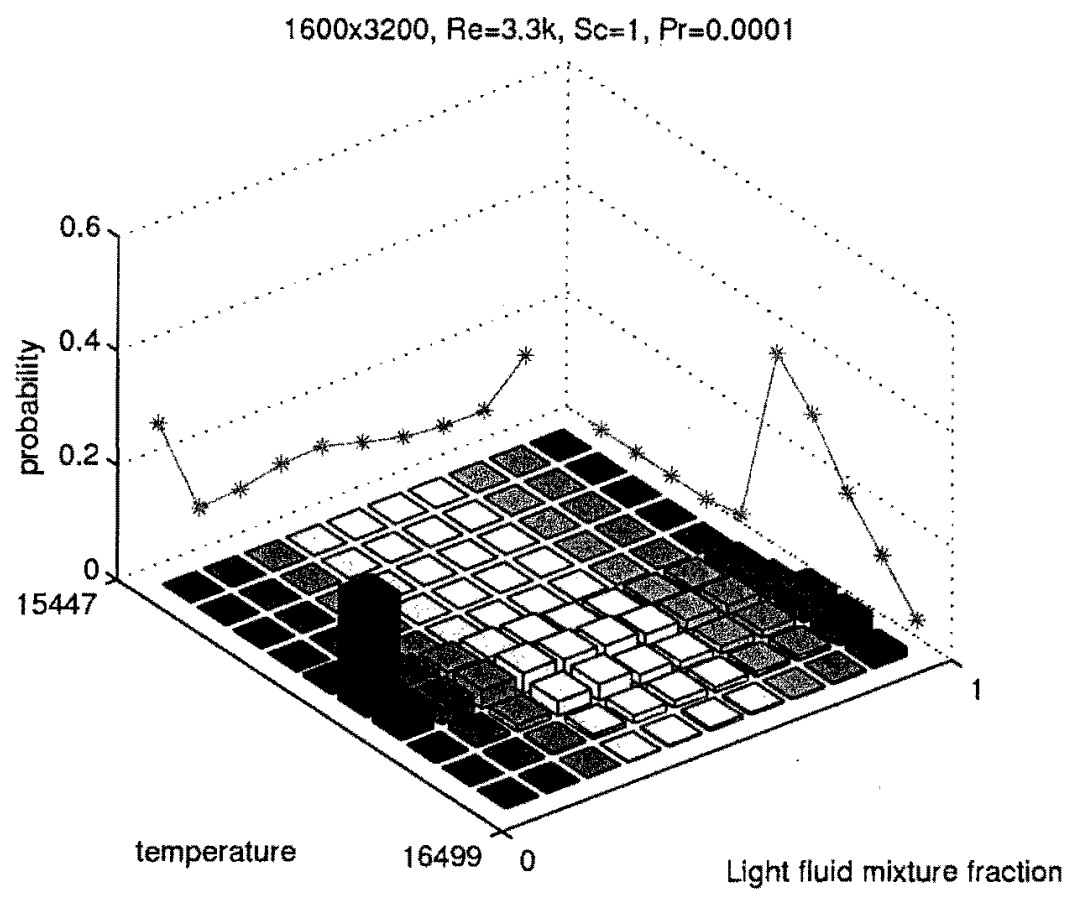

FIG. 9: Case p. The joint pdf of light species concentration and temperature at time $t=90$. The data has been collected into $10 \times 10$ bins. The mesh is $1600 \times 3200$ and $R e \approx 6000$.

The mean molecular mixing fraction $\theta$, defined ${ }^{29}$ as

$$
\theta=\frac{\left\langle f_{1} f_{2}\right\rangle}{\left\langle f_{1}\right\rangle\left\langle f_{2}\right\rangle}
$$

is a common measure of mixing. Here $f_{k}$ is the mass concentration fraction for the species $k$. In the DNS regime $\theta$ depends on the transport coefficients but is mesh independent. Perhaps the best way to understand $\theta$ is through its role in the chemical reaction production rate $w$ for a simple reaction $A+B \rightarrow C$, namely ${ }^{3}$

$$
w=\text { const. } f_{1} f_{2} \exp \left(-T_{A C} / T\right),
$$

where $T_{A C}$ is an activation temperature and $w$ is set to zero if $T<T_{A C}$. In this formula, we note that $\theta$ occurs naturally as a factor in the mean value $\langle w\rangle$ for $w$. As necessary, 


\begin{tabular}{|c|c|c|c|c|c|c|}
\hline & \multicolumn{2}{|c|}{$\mathrm{l}$} & \multicolumn{2}{|c|}{$\mathrm{g}$} & \multicolumn{2}{c|}{$\mathrm{p}$} \\
\hline$R e$ & $\mathrm{c}$ to $\mathrm{f}$ & $\mathrm{m}$ to $\mathrm{f}$ & $\mathrm{c}$ to $\mathrm{f}$ & $\mathrm{m}$ to $\mathrm{f}$ & $\mathrm{c}$ to $\mathrm{f}$ & $\mathrm{m}$ to $\mathrm{f}$ \\
\hline$\approx 300$ & 0.50 & 0.27 & 0.35 & 0.25 & 1.24 & 0.86 \\
$\approx 6000$ & 0.34 & 0.18 & 1.08 & 0.69 & 1.75 & 1.41 \\
$\approx 600 \mathrm{~K}$ & 1.02 & 0.56 & 0.98 & 0.46 & 0.49 & 0.34 \\
\hline
\end{tabular}

TABLE II: Mesh errors for the joint pdfs to illustrate possibilities of mesh convergence. Comparison is coarse (c) to fine (f) and medium ( $\mathrm{m}$ ) to fine. Various physical cases reported.

we will resort to moments $(\theta)$ to analyze the variability of the concentration, but due to the strongly nonlinear dependence of $w$ on $T$, we prefer to model $T$ using its pdf.

In order to compute the mean value fo $w$, we need to perform the statistical average defining $\theta$ at fixed $T$. This means that we bin together data points with $T$ in a common range (bin), and in this restricted ensemble, we compute means to define $\theta$. Let \langle\rangle$_{T}$ denote the expectation in the fixed $T$ spatial ensemble. It is defined as the sum of its argument over all sample points in a fixed $T$ bin divided by the number of sample points in that bin. The result is $\theta(T)$, as a function of temperature $T$, where

$$
\theta(T)=\frac{\left\langle f_{1} f_{2}\right\rangle_{T}}{\left\langle f_{1}\right\rangle_{T}\left\langle f_{2}\right\rangle_{T}}
$$

The result is shown in the nine frames of Fig. 10. We note that the gas case has values $\theta(T) \approx 0.5-0.65$ over most of the temperature range, and for the liquid case $\theta(T) \approx 0.45-0.5$ again over most of the temperature range. Aside from a peak $\theta(T)$ value associated with the lowest $T$ (caused by unmixed light fluid), most of the $\theta(T)$ range displays very little temperature dependence. Because the $\theta$ defined conventionally by a spatial average \langle\rangle (not constrained to a fixed $T$ value) as in (28) is distinct from $\theta(T)$, we include the $\theta$ value in each frame of Fig. 10 . We observe that $\theta \approx \theta(T)$, so the distinction between $\theta$ and $\theta(T)$ is primarily of technical significance. The $\theta$ values are generally lower than those obtained in other simulations for related problems. This fact reflects the elimination of numerical mass diffusion in our simulations and a greater occurance 


\begin{tabular}{|c|c|c|c|c|c|c|}
\hline & \multicolumn{2}{|c|}{$\mathrm{l}$} & \multicolumn{2}{c|}{$\mathrm{g}$} & \multicolumn{2}{c|}{$\mathrm{p}$} \\
\hline$\approx 300$ & 0.236 & 0.065 & 0.038 & 0.032 & 0.055 & 0.103 \\
$\approx 6000$ & 0.039 & 0.029 & 0.209 & 0.131 & 0.337 & 0.110 \\
$\approx 600 \mathrm{~K}$ & 0.135 & 0.068 & 0.145 & 0.060 & 0.094 & 0.032 \\
\hline
\end{tabular}

TABLE III: Mesh errors for $\theta(T)$. Comparison is coarse (c) to fine (f) and medium (m) to fine. Various physical cases reported.

of blobs of unmixed or poorly mixed fluid than is commonly observed numerically.

We supplement the visual convergence shown with a quantitative estimate of the error in $\theta(T)$. See Table III. Here the error is defined as

$$
e=\sum_{i=0}^{9} w_{i}\left|\theta_{i}^{c}-\theta_{i}^{f}\right|
$$

where $w_{i}$ is the weight of the corresponding temperature bin, calculated (for the coarse to fine comparison) as

$$
w_{i}=\frac{\sqrt{u_{i}^{c}} \sqrt{u_{i}^{f}}}{\sum_{i=0}^{9} \sqrt{u_{i}^{c}} \sqrt{u_{i}^{f}}}
$$

\section{Temperature PDF}

Encouraged by the fact that the variable $\theta(T)$ is mesh convergent (and largely independent of $T$ ), as a factor contributing to the chemistry production term $w$, we next take the step of analyzing the pdf for $T$. By definition, this pdf (which we denote $p(T)$ ), is the number of sample points in a $\mathrm{T}$ bin per degree size for the width of the bin in degrees Kelvin. We plot the pdf for $T$, in Fig. 11, for various physical cases.

Again the convergence properties are visually encouraging. Pursuing this point, we tabulate the errors for the pure $T$ pdf, to show grid convergence, see Table IV. The errors for the $T$ pdf are defined as in (31). Improvement over Table II should be noted. Observe that the underlying data has not changed, but only its mode of analysis. 

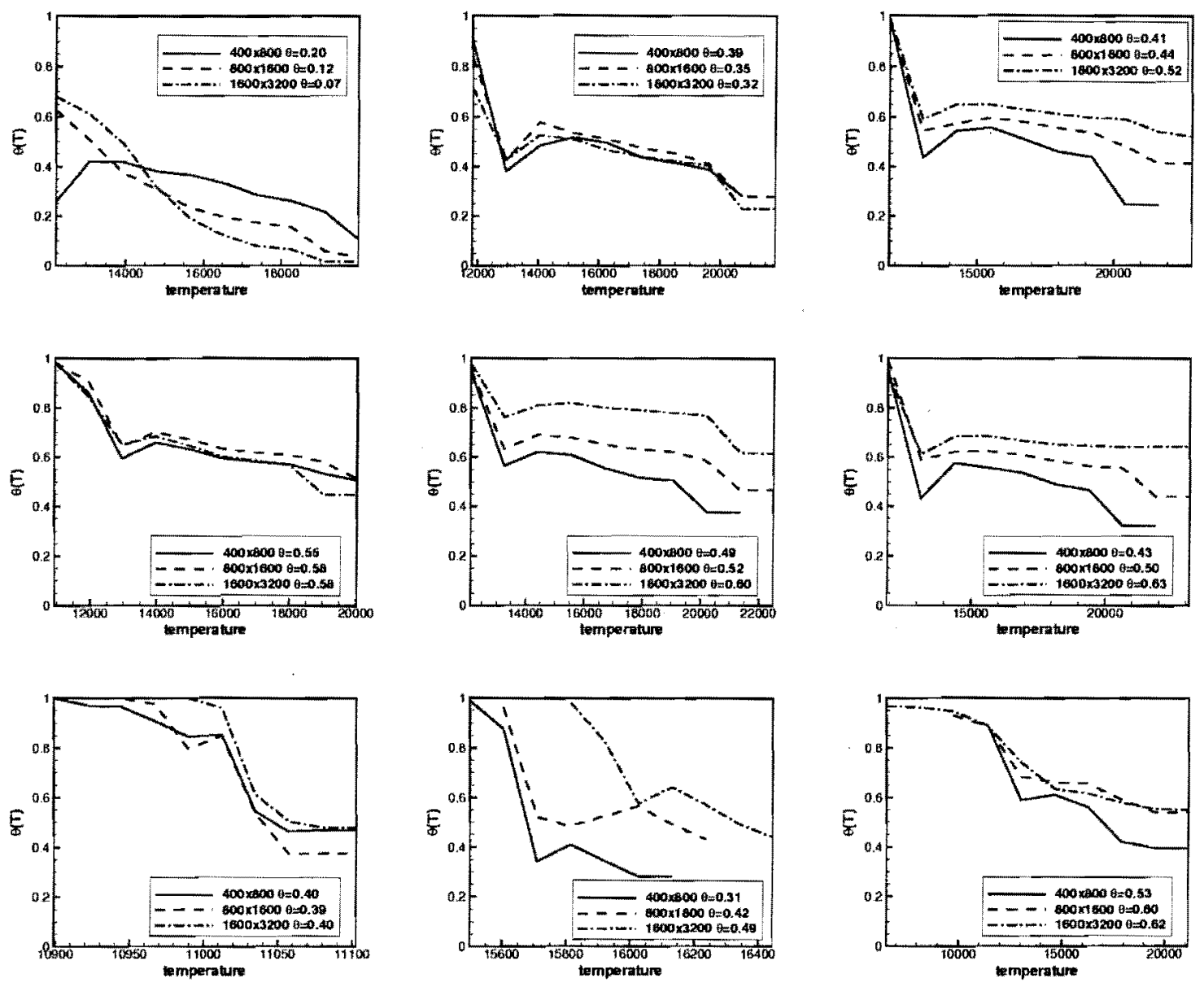

FIG. 10: Plot of $\theta(T)$ vs. $T$. Cases $1, \mathrm{~g}$, p (rows 1 to 3 ), with Re increasing, left to right.

We note an important distinction between the case $\mathrm{p}$ and the cases $\mathrm{l}$ and $\mathrm{g}$. For the high thermally diffusive case $p$, the range of temperatures spatially is very narrow. Accordingly, the convergence of the pdfs in this case is rather like convergence of near delta functions, and as the mesh is varied, there can even be only partial overlap in the temperature ranges observed. The Reynolds number dependence is significant in case $p$ but is very weak in cases 1 and $g$. 

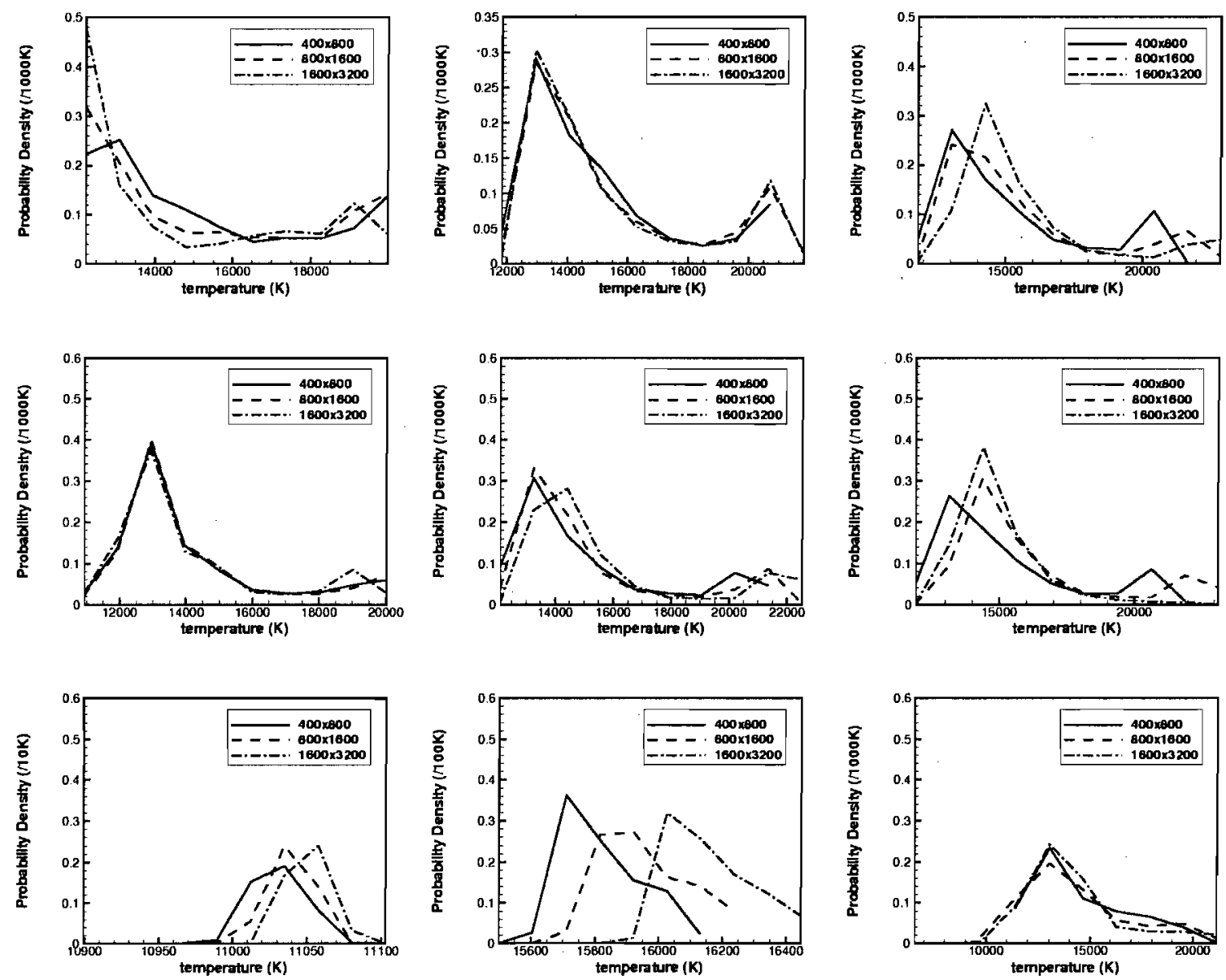

FIG. 11: Plot of $T$ pdf. Cases l, g, p (rows 1 to 3 ), with $R e$ increasing, left to right.

\section{Chemical Production}

Finally, we combine the separate analysis of $\theta(T)$ and $T$ to estimate a convergence rate for the mean chemical production term $\langle w\rangle$, assuming a fictious activation temperature $T_{A C}=10,000^{\circ} \mathrm{K}$.

Combining (30) and (29) we have

$$
\langle w\rangle=\int\langle w\rangle_{T} p(T) d T=\text { const. } \int\left\langle f_{1}\right\rangle_{T}\left\langle f_{2}\right\rangle_{T} \theta_{T} \exp \left(-T_{A C} / T\right) p(T) d T
$$

We list convergence properties for $\langle w\rangle$ in Table $\mathrm{V}$. We tabulate the relative error, de- 


\begin{tabular}{|c|c|c|c|c|c|c|}
\hline & \multicolumn{2}{|c|}{$\mathrm{l}$} & \multicolumn{2}{|c|}{$\mathrm{g}$} & \multicolumn{2}{c|}{$\mathrm{p}$} \\
\hline Re & $\mathrm{c}$ to $\mathrm{f}$ & $\mathrm{m}$ to $\mathrm{f}$ & $\mathrm{c}$ to $\mathrm{f}$ & $\mathrm{m}$ to $\mathrm{f}$ & $\mathrm{c}$ to f & $\mathrm{m}$ to $\mathrm{f}$ \\
\hline$\approx 300$ & 0.101 & 0.066 & 0.019 & 0.023 & 0.184 & 0.188 \\
$\approx 6000$ & 0.022 & 0.010 & 0.081 & 0.070 & 0.204 & 0.148 \\
$\approx 600 \mathrm{~K}$ & 0.137 & 0.094 & 0.151 & 0.060 & 0.035 & 0.048 \\
\hline
\end{tabular}

TABLE IV: Errors for the temperature pdfs shows mesh convergence. Comparison is coarse mesh (c) to fine (f) and medium (m) to fine. Various physical cases reported.

fined (for the coarse to fine comparison) as $\left|\left\langle w^{c}\right\rangle-\left\langle w^{f}\right\rangle\right| /\left\langle w^{f}\right\rangle$, where $w^{c}$ is the coarse grid production value $w$, etc. The convergence is very encouraging for an activation temperature $T_{A C}=10,000^{\circ} \mathrm{K}$ outside of the range of observed temperatures.

For the more difficult case of an activation temperature within the range of observed values, $w$ has a strong nonlinear dependence of $T$. We choose $T_{A C}=15,000^{\circ} \mathrm{K}$ to illustrate this case. The errors in this case are larger but still suggest convergence, and possibily the desirability of additional mesh refinement. But the somewhat erratic nature of the convergence suggests that the mean value $\langle w\rangle$ defined by a spatial average should be regarded as a random variable, with determination of an ensemble mean and variance. The case $R e=300$ for liquids remains exceptional. Because the temperature range in case $\mathrm{p}$ is so small, and varies (even to the point of being non-overlapping) as Re and the mesh is varied, it does not seem realistic to choose a $T_{A C}$ within the range of observed temperatures. Accordingly, we omit case p from Table VI. See Table VI.

\section{E. Statistical Fluctuation vs. Mesh Convergence}

Close examination of Table VI suggests anomalies with the convergence. For this reason we examine the mesh convergence more closely. First, we looked at errors or randomness associated with the binning process. The results did not show significant sensitivity to the binning. Next we examined the radial dependence of the convergence. 


\begin{tabular}{|c|c|c|c|c|c|c|}
\hline & \multicolumn{2}{|c|}{$\mathrm{l}$} & \multicolumn{2}{|c|}{$\mathrm{g}$} & \multicolumn{2}{|c|}{$\mathrm{p}$} \\
\hline$R e$ & $\mathrm{c}$ to $\mathrm{f}$ & $\mathrm{m}$ to $\mathrm{f}$ & $\mathrm{c}$ to $\mathrm{f}$ & $\mathrm{m}$ to $\mathrm{f}$ & $\mathrm{c}$ to $\mathrm{f}$ & $\mathrm{m}$ to $\mathrm{f}$ \\
\hline$\approx 300$ & 7.375 & 2.25 & 0.027 & 0.028 & 0.030 & 0.014 \\
$\approx 6000$ & 0.131 & 0.175 & 0.305 & 0.201 & 0.342 & 0.065 \\
$\approx 600 K$ & 0.308 & 0.147 & 0.291 & 0.092 & 0.194 & 0.043 \\
\hline
\end{tabular}

TABLE V: Relative mesh errors for the mean chemical production term $\langle w\rangle$ for an activation temperature $T_{A C}=10,000^{\circ} \mathrm{K}$. The comparison shows mesh convergence. Comparison is coarse mesh (c) to fine (f) and medium (m) to fine. Various physical cases reported.

\begin{tabular}{|c|c|c|c|c|c|c|}
\hline & \multicolumn{3}{|c|}{$\mathrm{l}$} & \multicolumn{3}{c|}{$\mathrm{g}$} \\
\hline$R e$ & $2 \sigma /\langle w\rangle$ & c to f & $\mathrm{m}$ to $\mathrm{f}$ & $2 \sigma /\langle w\rangle$ & $\mathrm{c}$ to $\mathrm{m}$ & $\mathrm{m}$ to $\mathrm{f}$ \\
\hline$\approx 300$ & 2.727 & 7.091 & 2.091 & 0.161 & 0.089 & 0.116 \\
$\approx 6000$ & 0.213 & 0.106 & 0.163 & 0.216 & 0.448 & 0.352 \\
$\approx 600 K$ & 0.128 & 0.441 & 0.195 & 0.286 & 0.221 & 0.273 \\
\hline
\end{tabular}

TABLE VI: Relative coarse grid ensemble fluctions $( \pm 2 \sigma)$ divided by ensemble mean, and relative mesh errors for the mean chemical production term $\langle w\rangle$ for an activation temperature $T_{A C}=15,000^{\circ} \mathrm{K}$. Comparison is coarse mesh (c) to fine (f) and medium (m) to fine. Only cases $\mathrm{I}$ and $\mathrm{g}$ are reported.

We found a significant radial dependence on the temperature, a consequence of the fact that the heavy material is hotter than the light. So the temperature and $w$, considered as a function of $r$, is biased towards the outer portion of the mixing zone. See Fig. 12. Finally, we considered the relative importance of mesh errors and statistical fluctuations. For the coarsest grid, and for cases $\mathrm{I}$ and $\mathrm{g}$ only, we introduced a modest ensemble (6 realizations) defined by randomly selected initial perturbations of the interface. We find two phenomena. When the mesh errors are very small, the random fluctuations (magnitude defined as $2 \sigma$ for this small ensemble) dominate, and the observed fluctuations 


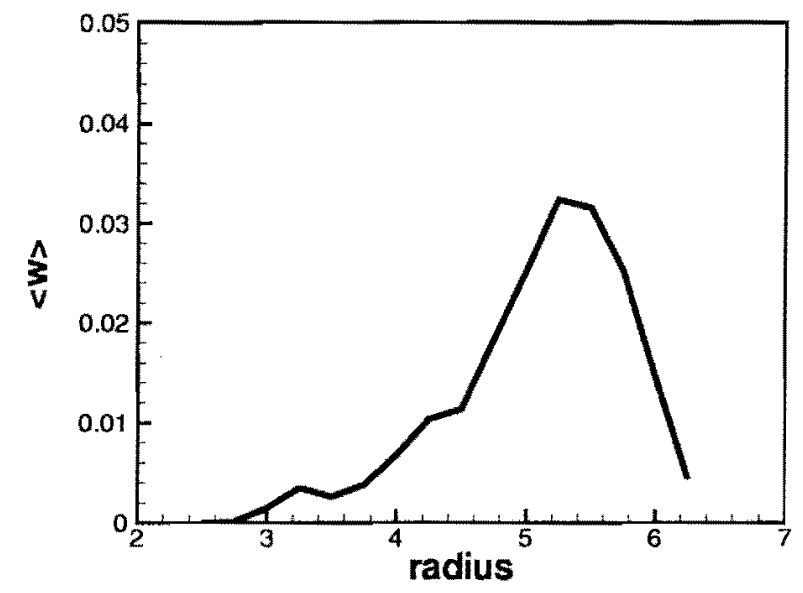

FIG. 12: Radial dependence for $\langle w\rangle$ for the case l, $R e \approx 6000$.

at a mesh level are probably chance events, rather than indicating convergence or its absence. When the mesh errors are larger, they dominate the statistical fluctuations, and apparently true mesh convergence is observed. See Table VI, where the coarse grid dimensionless (relative) fluctuations are tabulated under the heading $2 \sigma /\langle w\rangle$.

\section{DISCUSSION}

Our most important conclusion is the convergence for both macro and micro observables for a strongly chaotic mixing flow: reshocked Richtmyer-Meshkov instability. Mesh convergence for micro variables is partly obscured by statistical fluctuations, as noted below and in the body of the paper. New computational strategies and new methods of data analysis were required to reach this goal. We have combined (and enhanced) the resolution of sharp gradient near discontinuities of capturing codes with the subgrid physics modeling accuracy of turbulence codes. The convergence properties are basically uniform in Schmidt and Prandtl number. As far as the authors are aware, results of this nature have not been obtained by others.

A striking feature of the results is their relative independence on the Reynolds number, 
allowed to vary by a factor of 2000. Also significant is the strong dependence of the mixing properties of the simulation on the physical regimes with their distinct Schmidt and Prandtl numbers. THe impact of diffusion within the simulations is generally lower than commonly reported on the basis of under resolved untracked capturing codes. These codes substitute numerical transport properties for physical ones. The actual numerical transport values are not documented, but it is safe to guess that the effective numerical Schmidt and Prandtl numbers are mainly less than one, and thus more diffusive than the present gas case.

These trends in the diffusive properties of the solutions are predicted by the trends in the $\left(\lambda_{D}+\lambda_{D_{t}}\right) / \lambda_{C}$ and $\left(\lambda_{T}+\lambda_{T_{i}}\right) / \lambda_{C}$ ratios. In other words, elementary one dimensional diffusion analysis, coupled with the flow geometry, is sufficient to predict the major trends observed. We refer to the plot of these ratios in Figs. 6.

As new modes of data analysis, we have introduced the importance of convergence for the microscopic variables. These are subject to statistical fuctions which obscure mesh convergence. Some lavel of averaging is desirable, but we preserve important nonlinear functionals of the data, for which simple averaging is meaningless. This leads to the ensemble labeled by concentration and temperature values rather than by spatial coordinates. Using this framework and the typical observable of a chemical reaction rate, we observe a reduction of statistical fluctuation. Even after these steps; and for the specific problem considered, the relative size of mesh errors and fluctuations are in balance. But at least we can say that either the mesh errors are so small as to be insignificant or they are significant but dominate the fluctions and thereby mesh convergence can be observed.

\section{APPENDIX}

We develop the subgrid scale dynamic model terms used in this paper, following and slightly extending ideas of ${ }^{18,20}$. 


\section{A. A Subgrid Scale Dynamic Model for the Momentum Equation}

We use the trace-free Smagorinsky eddy viscosity model for the sub grid scale (SGS) stress $\tau_{i j}$. The $\tau_{i j}$ can be decomposed into an anisotropic (trace zero) tensor $\left(\tau_{i j}^{a}\right)$ and an isotropic tensor $\left(\tau_{i j}^{i}\right)$, which are modeled separately:

$$
\tau_{i j}^{M}=\left(\tau_{i j}-\tau_{k k} \frac{\delta_{i j}}{3}\right)+\tau_{k k} \frac{\delta_{i j}}{3}=\tau_{i j}^{a}+\tau_{i j}^{i}=-2 C_{S} \Delta^{2} \bar{\rho}|\widetilde{S}| \widetilde{S}_{i j}^{a}+\frac{\delta_{i j}}{3} 2 C_{I} \Delta^{2} \bar{\rho}|\widetilde{S}|^{2},
$$

where $\widetilde{S}_{i j}^{a}=\widetilde{S}_{i j}-\frac{\delta_{i j}}{3} \widetilde{S}_{k k}$. and $|\cdot|$ is defined by (4). The $C_{S}$ and $C_{I}$ are model coefficients to be computed dynamically. In analogy to (21), we define

$$
\nu_{k t}=C_{S} \Delta^{2} \overline{|\widetilde{S}|}
$$

as the kinematic turbulent viscosity.

The key element of the dynamic model is the utilization of the data contained in the resolved field. This information is brought to bear by introducing a test filter with a larger filter width $\widehat{\Delta}$ than the resolved grid filter. We will use a $2 \times 2$ mesh block average to define the test filter. Let a spatially test-filtered quantity be denoted by a caret. The test filtered stress $T_{i j}$ is defined as:

$$
T_{i j}=\widehat{\overline{\rho v_{i} v_{j}}}-\frac{\widehat{\overline{\rho v_{i}}} \widehat{\widehat{\rho v_{j}}}}{\widehat{\bar{\rho}}}
$$

and is modeled as:

$$
T_{i j}^{M}=T_{i j}^{u}+T_{i j}^{i}=-2 C_{S} \widehat{\Delta}^{2} \widehat{\bar{\rho}}|\widehat{\widetilde{S}}| \widehat{\widetilde{S}}_{i j}^{a}+\frac{2 \delta_{i j}}{3} C_{I} \widehat{\Delta}^{2} \widehat{\bar{\rho}}|\widehat{\widetilde{S}}|^{2}
$$

Using Germano's identity ${ }^{8}$, the Leonard stress $L_{i j}$ can be expressed as:

$$
L_{i j}=T_{i j}-\widehat{\tau_{i j}}=\left(\frac{\widehat{\rho v_{i}} \overline{\rho v_{j}}}{\bar{\rho}}\right)-\frac{\widehat{\rho v_{i}} \widehat{\rho v_{j}}}{\widehat{\widehat{\rho}}}=\widehat{\bar{\rho} \widetilde{v}_{i} \widetilde{v}_{j}}-\frac{\widehat{\bar{\rho} v_{i}} \widehat{\widetilde{\rho} v_{j}}}{\widehat{\bar{\rho}}}
$$

The right hand side is completely computable from the resolved variables. We next introduce the ansatz that $C_{S}$ and $C_{I}$ are independent of the length scale. In other words, the same $C_{S}$ and $C_{I}$ occur in (34) and (37). In this case,

$$
L_{i j}^{a}=T_{i j}^{u}-\widehat{\tau}_{i j}^{a}=2 C_{S} \Delta^{2} \bar{\rho}\left|\widetilde{S^{\prime} \mid \widetilde{S}_{i j}^{a}}-2 C_{S} \widehat{\Delta}^{2} \widehat{\bar{\rho}}\right| \widehat{\widetilde{S}} \mid \widehat{\widetilde{S}}_{i j}^{a}=C_{S} M_{i j}^{a}
$$


where

$$
M_{i j}^{a}=2 \Delta^{2} \overline{\hat{\rho}|\widetilde{S}| \widetilde{S}_{i j}^{a}}-2 \widehat{\Delta}^{2} \widehat{\hat{\rho}}|\widehat{\widetilde{S}}| \widehat{\widetilde{S}}_{i j}^{a}
$$

We would next solve (39) for $C_{S}$. However, this equation corresponds to five independent relations for $C_{S}$ and an algebraic solution is not possible.

We introduce an averaging operation $\langle\cdots\rangle$. The specification of the average is problem dependent, as the universal definition of an ensemble average is inconvenient to use. For the present problem, we regard this average as taken over the symmetry variable $\theta$, i.e., an average over circular arcs (constant radius). To assure numerical regularity, we also apply a convolution average in the radial direction with a stencil extending $\pm 6 \Delta r$ from current radius.

Applying this average to (39) and (40) and using least squares in the resulting equations leads to the formula

$$
C_{S}=\frac{\left\langle\left(\sum L_{i j}^{a} M_{i j}^{a}\right)^{+}\right\rangle}{\left\langle\left(\sum M_{i j}^{a} M_{i j}^{a}\right)^{+}\right\rangle} .
$$

Here the expression $(\cdots)^{+}$means the positive part of the quantity in the parenthesis. In other words, we clip negative values. This step is consistent with methods proposed elsewhere $^{18}$. The same method is used for the coefficients of turbulent heat conduction and species concentration diffusion.

The turbulent viscosity determined by (41) and the related turbulent transport coefficients determined below were monitored for the simulations of the present study. The results were generally consistent with theoretical expectations. For example, the turbulent viscosity was respectively small (comparable, dominant) in relation to the molecular value of viscosity for the three cases of DNS, LES near to DNS, and LES far from DNS.

To determine $C_{I}$, we consider

$$
L_{k k}^{i}=T_{k k}^{i}-\widehat{\tau}_{k k}^{i}=-2 C_{I} \Delta^{2} \widehat{\bar{\rho}|\widetilde{S}|^{2}}+2 C_{I} \widehat{\Delta}^{2} \widehat{\bar{\rho}}|\widehat{\widetilde{S}}|^{2}=C_{I} M_{k k}^{i}
$$

where a summation convention was used for the $k k$ repeated indices and

$$
M_{k k}^{i}=-2 \Delta^{2} \overline{\bar{\rho}|\widetilde{S}|^{2}}+2 \widehat{\Delta}^{2} \widehat{\bar{\rho}}|\widehat{\widetilde{S}}|^{2}
$$


This equation corresponds to one relation for $C_{T}$, from which we obtain

$$
C_{I}=\frac{\left\langle L_{k k}^{i}\right\rangle}{\left\langle M_{k k}^{i}\right\rangle}
$$

To discretize the elliptic operators associated with the molecular level and subgrid scale transport terms, we use the same stencil at each front point that was used in the front propagation. This stencil is rectilinear, and aligned with the normal and tangential directions to the front at the front point in question. We use three mesh points in each of the normal and the tangential directions. Letting $\partial_{N}$ and $\partial_{T}$ denote derivatives in the normal and tangential directions, we first write the discretization for $\partial_{N} c \partial_{N} f$ for some function $f$ and spatially dependent coefficient $c$. The function is double valued at the front, and so we specify, as an example, a discretization of the left value, $f_{l}$. The discrete operator has the form

$$
c_{+1 / 2}\left(f_{+}-f_{0 l}\right)-c_{-1 / 2}\left(f_{0 l}-f_{-}\right)=c_{+1 / 2} f_{+}-\left(c_{+1 / 2}+c_{-1 / 2}\right) f_{0 l}+c_{-1 / 2} f_{-} .
$$

Here $f_{0 l}$ and $f_{0 r}$ are front states on the left and right side of the front, while $f_{ \pm}$and $c_{ \pm 1 / 2}$ are evaluated along the normal to the front at distances $\pm \Delta x$ or $\pm 1 / 2 \Delta x$ from the front.

The normal direction discretization for the right front state is similar, and the tangential direction discretizations differ only in that all values are associated with either the left or the right side of the front.

The discretization of the mixed partials is complicated by the use of a 5 point stencil and a fixed order of differentiation. We write

$$
\partial_{N} c \partial_{T} f=\partial_{T} c \partial_{N} f+\left(\partial_{N} c\right)\left(\dot{\partial}_{T} f\right)-\left(\partial_{T} c\right)\left(\partial_{N} f\right)
$$

The normal sweep is performed in advance of the tangential sweep. Use of (46) allows all mixed partial derivatives to be evaluated in the order of the sweeps, first normal derivatives and then tangential derivatives. During the normal sweep, we evaluate and store front values for $\partial_{N} c$ and $\partial_{N} f$, which can be differenced using obvious formulas during the tangential sweep to yield a discrete expression for the LHS of (46). 


\section{B. A Subgrid Scale Dynamic Model for the Energy Equation}

We only consider the eddy diffusivity SGS model for the SGS heat transport flux $q_{i}^{(H)}$ with some modeling assumptions (e.g. negligible subgrid viscous work and triple correlations). As in ref. ${ }^{12}$, we set $q_{i}^{(T)}=0=q_{i}^{(V)}$. The SGS heat transport flux is modeled as:

$$
\begin{gathered}
q_{i}^{(H) M}=-\bar{\rho} \widetilde{c_{p}} \frac{C_{S} \Delta^{2}|\widetilde{S}|}{P r_{t}} \frac{\partial \widetilde{T}}{\partial x_{i}} \\
=-\frac{\widetilde{c_{p}} \nu_{t}}{P r_{t}} \frac{\partial \widetilde{T}}{\partial x_{i}}=-\kappa_{t} \frac{\partial \widetilde{T}}{\partial x_{i}},
\end{gathered}
$$

where $P r_{t}$ is the SGS turbulent Prandtl number to be determined using a dynamic model.

At the test filter level, the flux is defined as:

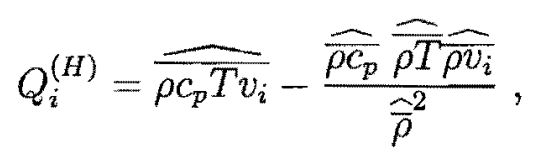

and is modeled as:

$$
Q_{i}^{(H) M}=-\widehat{\bar{\rho}} \widehat{\widetilde{c_{p}}} \frac{C_{S} \widehat{\Delta}^{2}|\widehat{\widetilde{S}}|}{P r_{t}} \frac{\partial \widetilde{\widetilde{T}}}{\partial x_{i}} .
$$

Again, using Germano's identity, we have

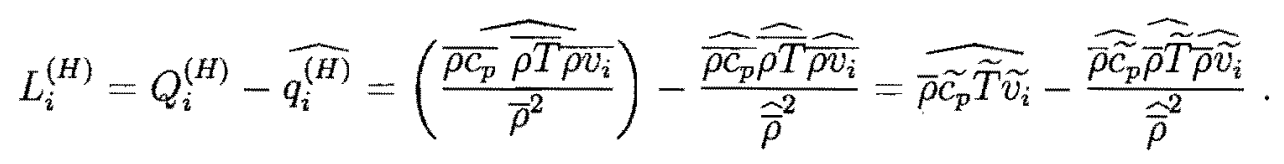

To determine $P r_{t}$

$$
L_{i}^{(H)}=Q_{i}^{(H) M}-\widehat{q_{i}^{(H) M}}=\frac{C_{S}}{P r_{t}}\left(\Delta^{2}\left(\bar{\rho} \widetilde{\rho} \widetilde{p}_{p}|\widetilde{S}| \frac{\partial \widetilde{T}}{\partial x_{i}}\right)-\widehat{\Delta}^{2} \widehat{\bar{\rho}} \widehat{\widetilde{c_{p}}}|\widehat{\widetilde{S}}| \frac{\partial \widetilde{\widetilde{T}}}{\partial x_{i}}\right)=\frac{C_{S}}{P r_{t}} M_{i}^{(H)}
$$

where

$$
M_{i}^{(H)}=\Delta^{2}\left(\bar{\rho} \widetilde{\rho} \widetilde{c_{p}}|\widetilde{S}| \frac{\partial \widetilde{T}}{\partial x_{i}}\right)-\widehat{\Delta}^{2} \widehat{\bar{\rho}} \widehat{\widetilde{c_{p}}}|\widehat{\widetilde{S}}| \frac{\partial \widetilde{\widetilde{T}}}{\partial x_{i}}
$$


This equation corresponds to three independent relations and again a least square approach is followed to calculate the model coefficient,

$$
\operatorname{Pr}_{t}=C_{S} \frac{\left\langle\left(\sum M_{i}^{(H)} M_{i}^{(H)}\right)^{+}\right\rangle}{\left\langle\left(\sum L_{i}^{(H)} M_{i}^{(H)}\right)^{+}\right\rangle} .
$$

Discretization is as in (45).

\section{A Subgrid Scale Dynamic Model for the Concentration Equation}

For SGS scalar transport, from a gradient transport modeling assumption,

$$
q_{i}^{(\psi) M}=-\bar{\rho} \frac{C_{S} \Delta^{2}|\widetilde{S}|}{S c_{t}} \frac{\partial \widetilde{\psi}}{\partial x_{i}}=-\frac{\nu_{d t}}{S c_{t}} \frac{\partial \widetilde{\psi}}{\partial x_{i}}
$$

where $S c_{t}$ is the SGS turbulent Schmidt number to be determined using a dynamic model.

At the test filter level, the SGS scalar transport is defined as:

$$
Q_{i}^{(\psi)}=\widehat{\overline{\rho v_{i} \psi}}-\frac{\widehat{\rho v_{i}} \widehat{\overline{\rho \psi}}}{\widehat{\bar{\rho}}}
$$

and is modeled as:

$$
Q_{i}^{(\psi) M}=-\widehat{\bar{\rho}} \frac{C_{S} \widehat{\Delta}^{2}|\widehat{\widetilde{S}}|}{S c_{t}} \frac{\partial \widetilde{\psi}}{\partial x_{i}}
$$

Using Germano's identity, we have

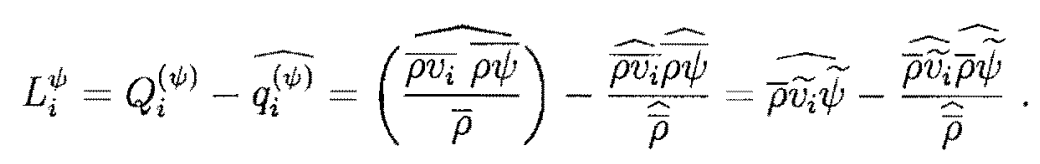

To determine $S c_{t}$

$$
L_{i}^{\psi}=Q_{i}^{(\psi) M}-\widehat{q_{i}^{(\psi) M}}=\frac{C_{S}}{S c_{t}}\left(\Delta^{2}\left(\overline{\bar{\rho}|\widetilde{S}| \frac{\partial \widetilde{\psi}}{\partial x_{i}}}\right)-\widehat{\Delta}^{2} \widehat{\bar{\rho}}|\widehat{\widetilde{S}}| \frac{\partial \widetilde{\tilde{\psi}}}{\partial x_{i}}\right)=\frac{C_{S}}{S c_{t}} M_{i}^{(\psi)}
$$

where

$$
M_{i}^{(\psi)}=\Delta^{2}\left(\bar{\rho}|\widetilde{S}| \frac{\partial \widetilde{\psi}}{\partial x_{i}}\right)-\widehat{\Delta}^{2} \widehat{\bar{\rho}}|\widehat{\widetilde{S}}| \frac{\partial \widehat{\widetilde{\psi}}}{\partial x_{i}}
$$


This equation corresponds to three independent relations. Again, a least square approach is followed to calculate the model coefficient,

$$
S c_{t}=C_{S} \frac{\left\langle\left(\sum M_{i}^{(\psi)} M_{i}^{(\psi)}\right)^{+}\right\rangle}{\left\langle\left(\sum L_{i}^{(\psi)} M_{i}^{(\psi)}\right)^{+}\right\rangle}
$$

Discretization is as in (45).

* This work was supported in part by U.S. Department of Energy grants DE-AC02-98CH10886 and DE-FG52-06NA26208, and the Army Research Office grant W911NF0510413. The simulations reported here were performed in part on the Galaxy linux cluster in the Department of Applied Mathematics and Statistics, Stony Brook University, and in part on New York Blue, the BG/L computer operated jointly by Stony Brook University and BNL. Los Alamos National Laboratory Preprint, LA-UR-XX-YYY.

1 K. Asano. Mass Transfer: From Fundamentals to Modern Industrial Applications. WileyVCH, 2006.

2 T. Barberon and P. Helluy, Finite volume simulation of cavitating flow. Computers and Fluids, 34:832-858, 2005 .

${ }^{3}$ R. S. Cany and K. Mastorakos. An Introduction to Turbulent Reacting Flows. Imperial College Press, London, 2008.

4 P. Colella. A direct Eulerian MUSCL scheme for gas dynamics. SIAM journal on scientific computing, 6(1):104-117, 1985.

5 Paul Drake. Hign energy Density Physics: Fundamentals, Inertial Fusion, and Experimental Astrophysics (Shock Wave and High Pressure Phenomena). Springer Verlag, 2006.

6 Jian Du, Brian Fix, James Glimm, Xicheng Jia, Xiaolin Li, Yunhua Li, and Lingling Wu. A simple package for front tracking. J. Comput. Phys., 213:613-628, 2006. Stony Brook University preprint SUNYSB-AMS-05-02.

7 S. Dutta, E. George, J. Glimm, J. Grove, H. Jin, T. Lee, X. Li, D. H. Sharp, K. Ye, Y. Yu, Y. Zhang, and M. Zhao. Shock wave interactions in spherical and perturbed spherical 
geometries. Nonlinear Analysis, 63:644-652, 2005. University at Stony Brook preprint number SB-AMS-04-09 and LANL report No. LA-UR-04-2989.

8 M. Germano, U. Piomelli, P. Moin, and W. H. Cabot. A dynamic subgrid scale eddy viscosity model. Phys Fluids A, 3:1760-1765, 1991.

9 J. Glimm, J. W. Grove, Y. Kang, T. Lee, X. Li, D. H. Sharp, Y. Yu, K. Ye, and M. Zhao. Statistical Riemann problems and a composition law for errors in numerical solutions of shock physics problems. SISC, 26:666-697, 2004. University at Stony Brook Preprint Number SBAMS-03-11, Los Alamos National Laboratory number LA-UR-03-2921.

10 J. Glimm, J. W. Grove, X. L. Li, W. Oh, and D. H. Sharp. A critical analysis of RayleighTaylor growth rates. J. Comput. Phys., 169:652-677, 2001.

11 J. Glimm, D. Marchesin, and O. McBryan. A numerical method for two phase flow with an unstable interface. J. Comput. Phys., 39:179-200, 1981.

12 D. J. Hill, C. Pantano, and D. L. Pullin. Large-eddy simulation and multiscale modeling of a Richtmyer-Meshkov instability with reshock. J. Fluid Mech, 557:29-61, 2006.

13 R. L. Holmes, B. Fryxell, M. Gittings, J. W. Grove, G. Dimonte, M. Schneider, D. H. Sharp, A. Velikovich, R. P. Weaver, and Q. Zhang. Richtmyer-Meshkov instability growth: Experiment, simulation, and theory. J. Fluid Mech., 389:55-79, 1999. LA-UR-97-2606.

14 M. M. Koochesfahani and P. E. Dimotakis. Mixing and chemical reactions in a turbulent liquid layer. J. Fluid Mech., 170:83-112, 1986.

15 H. Lim, Y. Yu, J. Glimm, X.-L. Li, and D. H. Sharp. Chaos, transport, and mesh convergence for fluid mixing. Acta Mathematicae Applicatae Sinica, 24:355-368, 2008. Stony Brook University Preprint SUNYSB-AMS-07-09 Los Alamos National Laboratory preprint number LA-UR-08-0068.

${ }^{16}$ H. Lim, Y. Yu, H. Jin, D. Kim, H. Lee, J. Glimm, X.-L. Li, and D. H. Sharp. Multi scale models for fluid mixing. Compu. Methods Appl. Mech. Engrg., 197:3435-3444, 2008. Stony Brook University Preprint SUNYSB-AMS-07-05.

17 X. F. Liu, Y. H. Li, J. Glimm, and X. L. Li. A front tracking algorithm for limited mass 
diffusion. J. of Comp. Phys., 222:644-653, 2007. Stony Brook University preprint number SUNYSB-AMS-06-01.

18 T. Ma. Large eddy simulation of variable density flows. Ph.d. thesis, University of Maryland, 2006 .

19 T. O. Masser. Breaking Temperature Equilibrium in Mixed Cell Hydrodynamics. Ph.d. thesis, State University of New York at Stony Brook, 2007.

20 P. Moin, K. Squires, W. Cabot, and S. Lee. A dynamic subgrid-scale model for compressible turbulence and scalar transport. Phys. Fluids, A3:2746-2757, 1991.

21 Heintz Pitsch. Large-eddy simulation of turbulent combustion. Annual Rev. Fluid Mech., $38: 453-482,2006$.

22 A. Polyanin. Hydrodynamics, Mass and Heat Transfer in Chemical Engineering, volume 14 of Topics in Chemical Engineering. CRC Press, 2002.

23 G. C. Pomraning. Linear kinetic theory and particle transport in stochastic mixtures, volume 7 of Series on Advances in Mathematics for Applied Sciences. World Scientific, Singapore, 1991.

24 G. C. Pomraning. Transport theory in discrete stochastic mixtures. Advances in Nuclear Science and Technology, 24:47-93, 1996.

${ }^{25}$ H. F. Robey. The effect of viscosity and mass diffusion in hydrodynamically unstable plasma flows. Phys. of Plasmas, 11:4123-4133, 2004.

${ }^{26}$ C. Thomas, S. Kumar, G. Orlicz, and K. Prestridge. An experimental investigation of mixing mechanisms in shock-accelerated flow, J. Fluid Mech., 611:131-150, 2008.

27 Forman Williams. Combustion Theory. Addison-Wesley Co., Reading, 1965.

28 P. Woodward and P. Colella. Numerical simulation of two-dimensional fluid flows with strong shocks. J. Comput. Phys., 54:115, 1984.

29 D. L. Youngs. Three-dimensional numerical simulation of turbulent mixing by RayleighTaylor instability. Phys. Fluids A, 3:1312-1319, 1991.

${ }^{30}$ Y. Yu, M. Zhao, T. Lee, N. Pestieau, W. Bo, J. Glimm, and J. W. Grove. Uncertainty 
quantification for chaotic computational fluid dynamics. J. Comput. Phys., 217:200-216, 2006. Stony Brook Preprint number SB-AMS-05-16 and LANL preprint number LA-UR05-6212. 